\title{
تهليل الاثر الارتدادي والتفاعلي بين السياسة المالية والنقدية على التوازن الاقتصادي العام ( IS-LM )
}

\author{
م. م. باسم خميس عبيد \\ أ.م.د. فريد جواد كاظم الاليمي

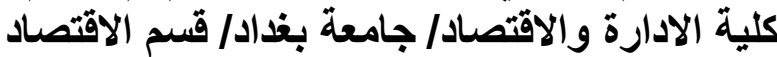

basiem1977@coadec.uobaghdad.edu.iq $\quad \underline{\text { Fareed_jawad@coadec.uobaghdad.edu.iq }}$

يتنـاول هذا البحث بالتحليل التفاعل والتنسيق بين السياستين الماليـة والنقايـة واثر هذا التئة التفاعل

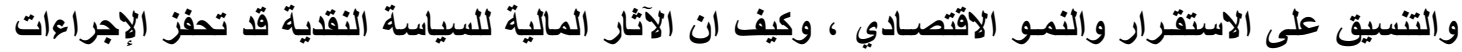

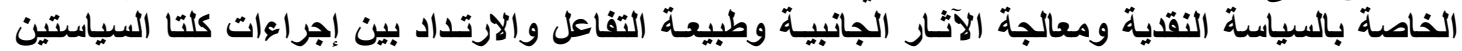

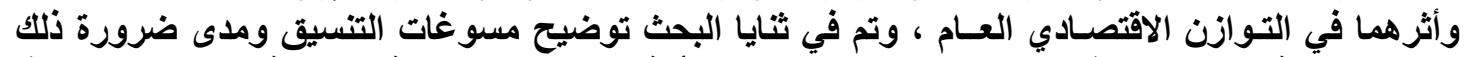

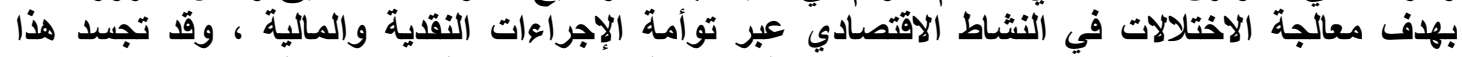

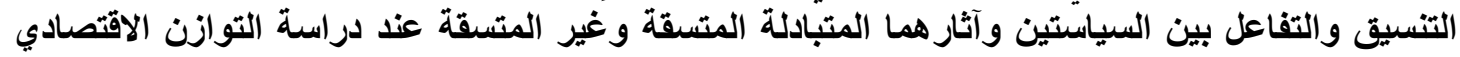

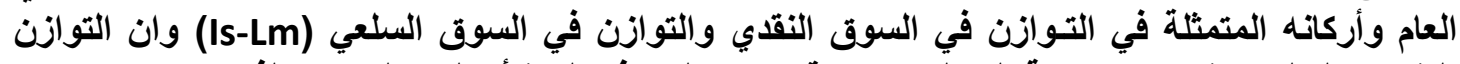

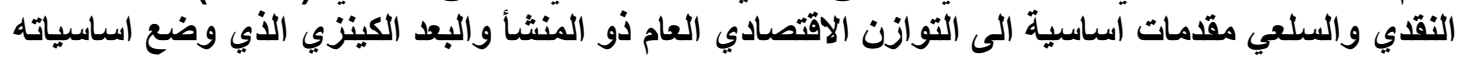

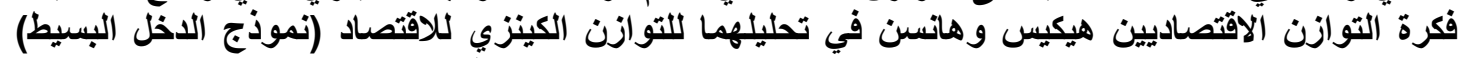

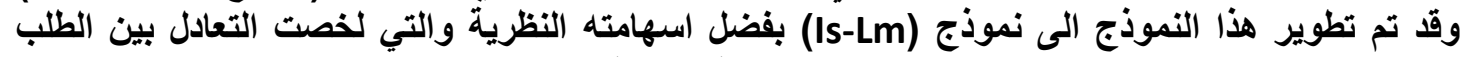

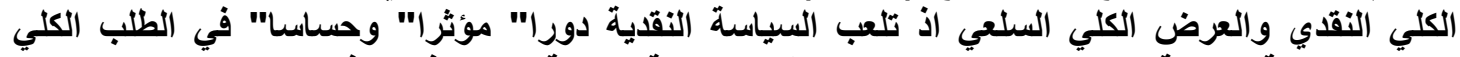

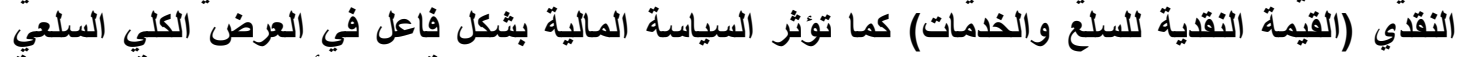

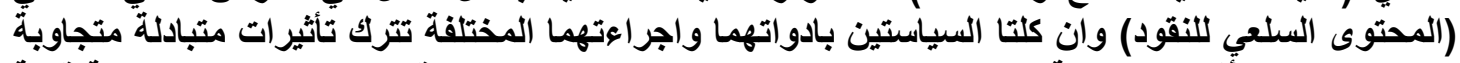

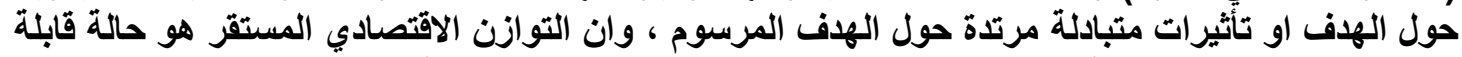

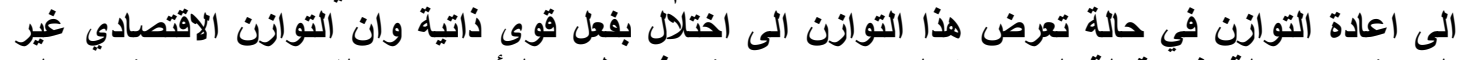

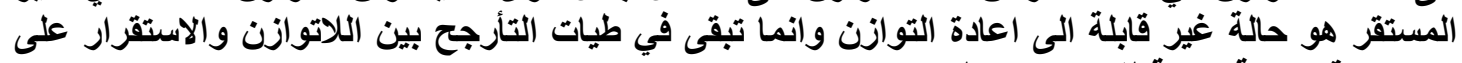

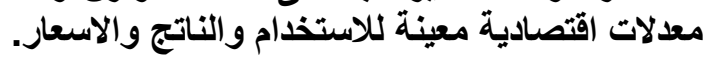

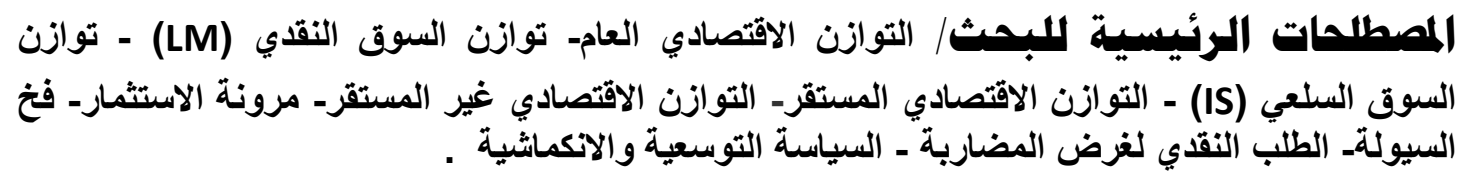

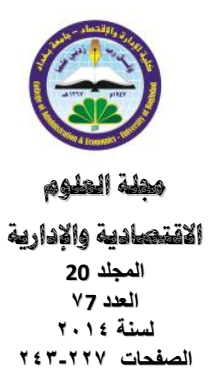

*ملاحظة : البحث مستل من اطروحة دكتوراه 
لم يحظ موضوع في علم الاقتصاد والفكر الاقتصادي بالدراسة والتقصي قدر ما حظي به موضوع

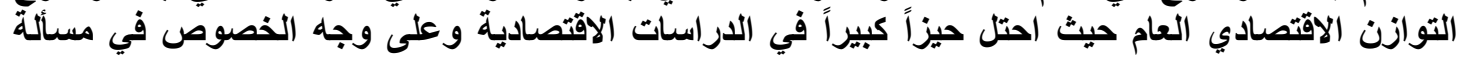

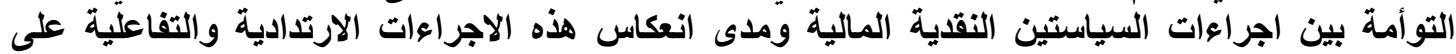

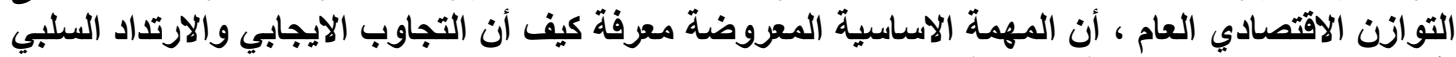

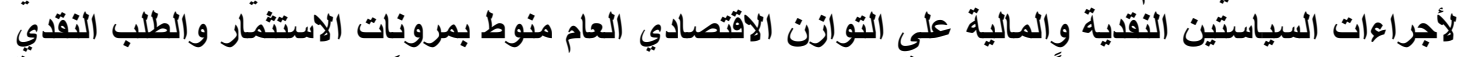

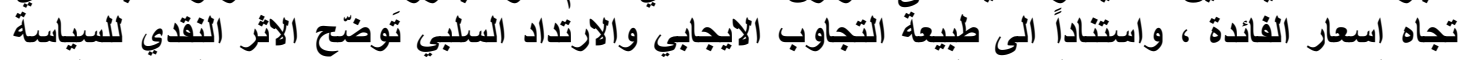

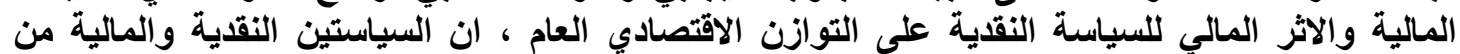

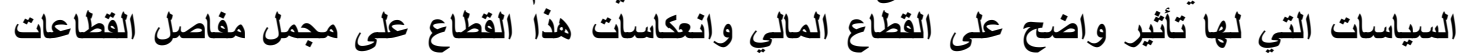

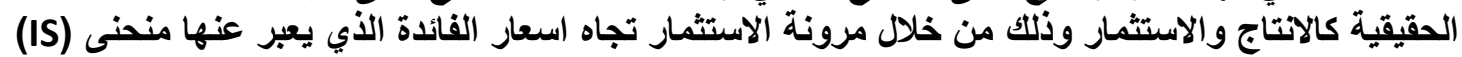

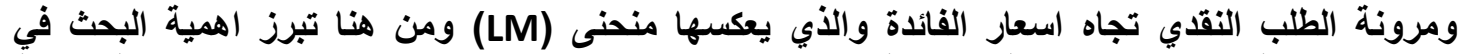

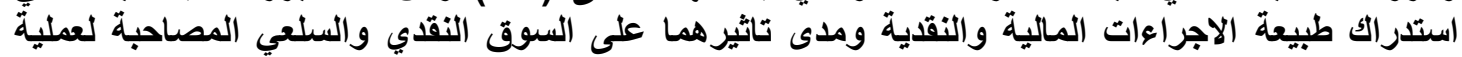

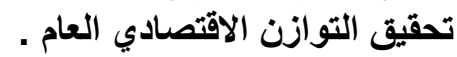

هشكاة البحث : ان عدم التناست بين اجراعت السياستين النقدية والمالية تخلق حالة عدم الاستقرار

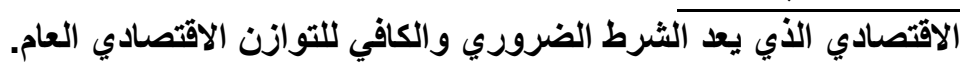

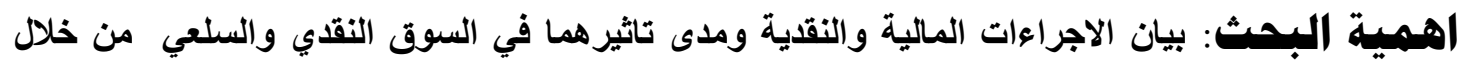
مرونة الاستثمار ومرونة الطلب النقدي تجاه اسعار الفائدة.

هيكاية البحث : وبهدف التحقق من صحة فرضية البحث تم تقيم البحث على ثلاث مباحث اذ تطرق

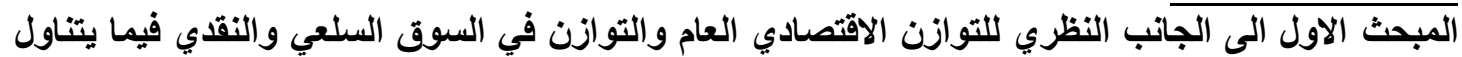

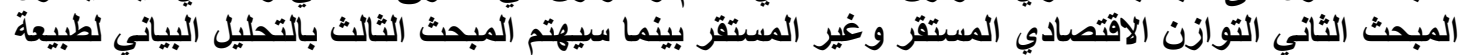

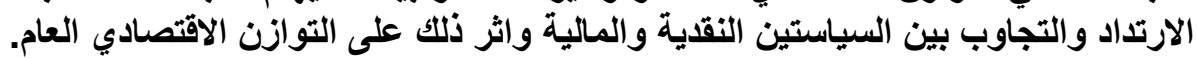

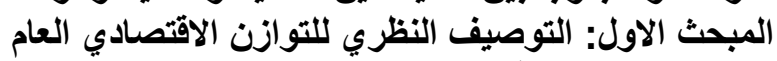

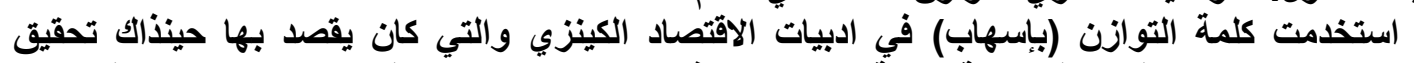

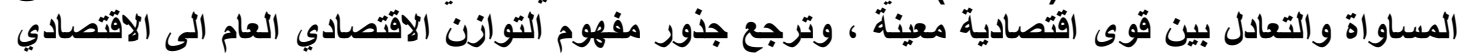

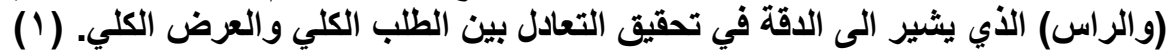

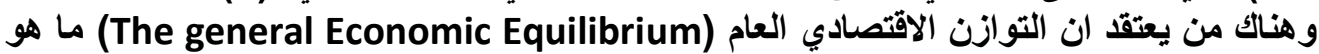

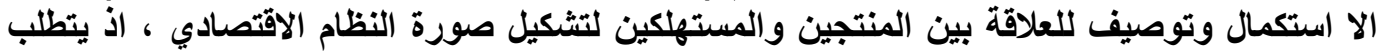

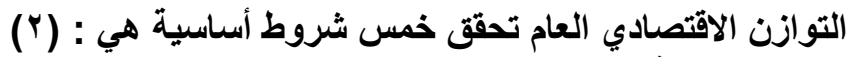

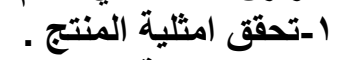

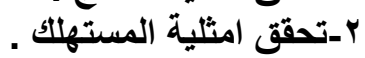

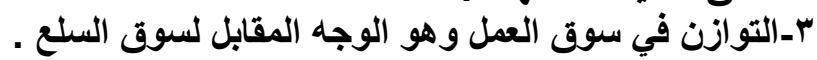

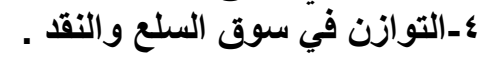

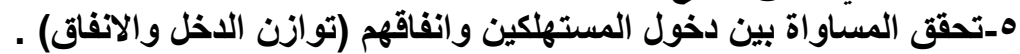

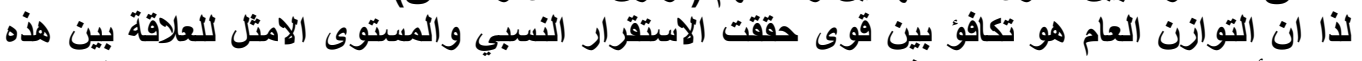

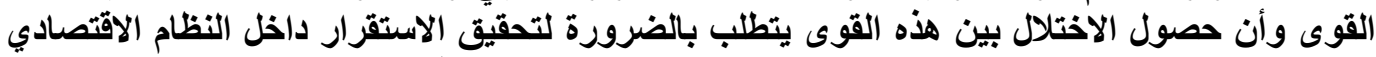

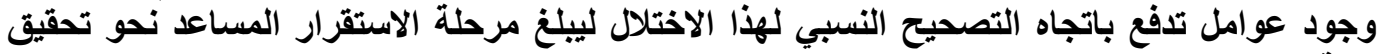


وان اي تغير في المستويات التوازنية للاسواق في الاقتصاد تسبب تغيرات إتهات اضافية تثابه

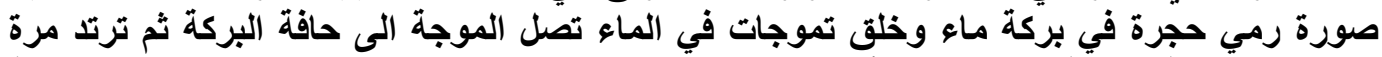

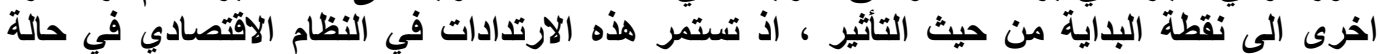

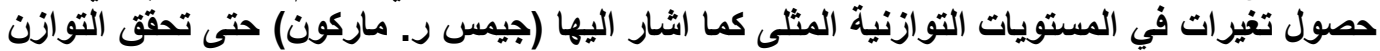

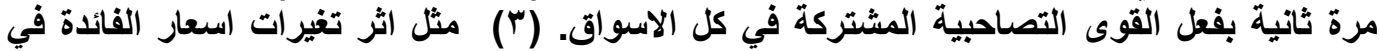

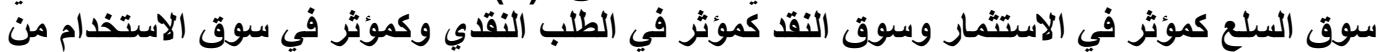

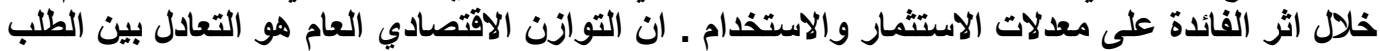

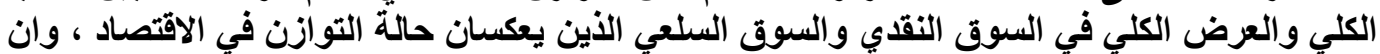

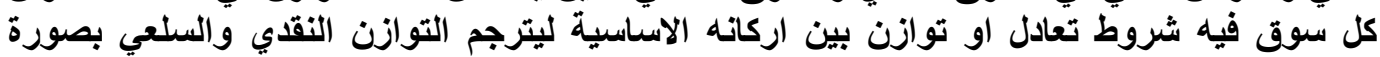

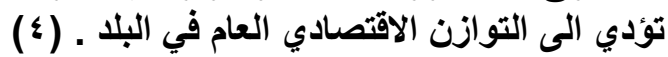

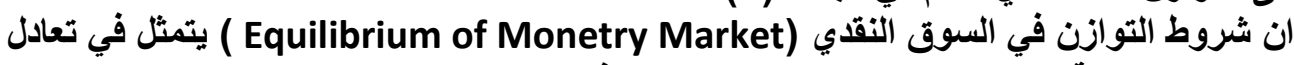

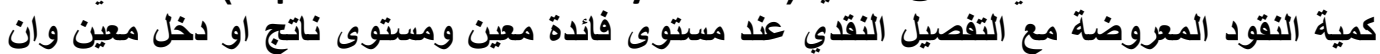

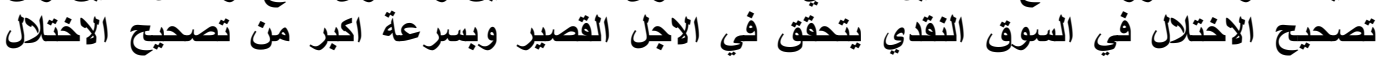

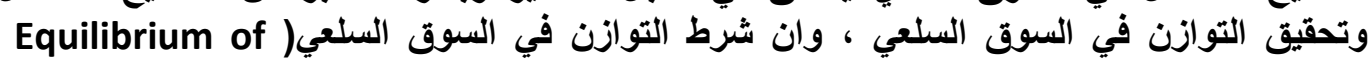

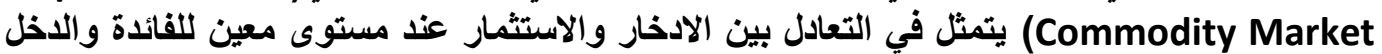

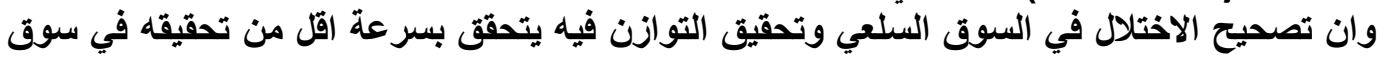

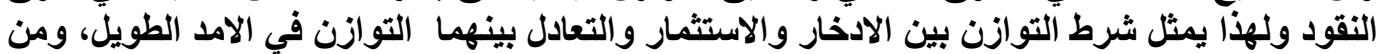

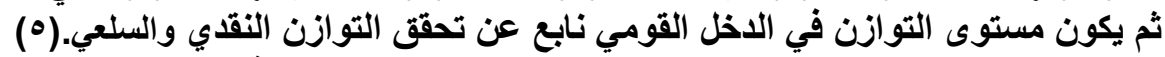

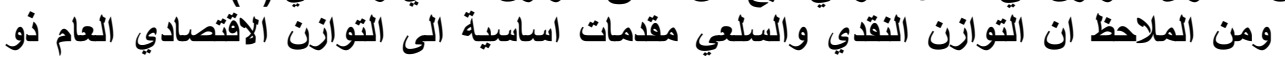

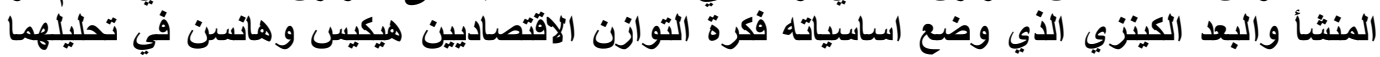

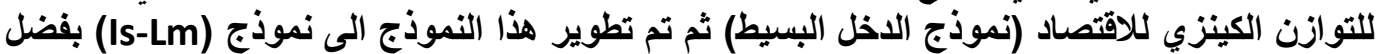

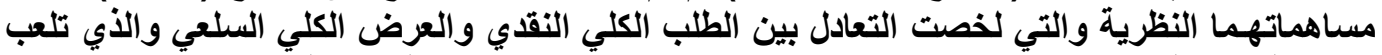

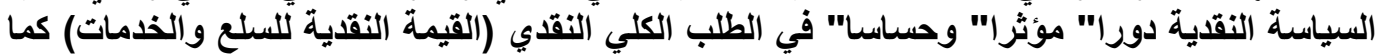

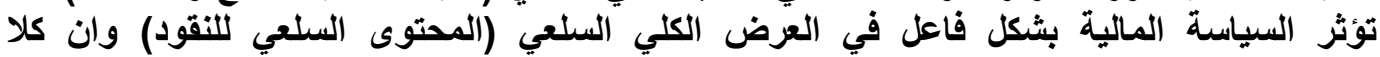

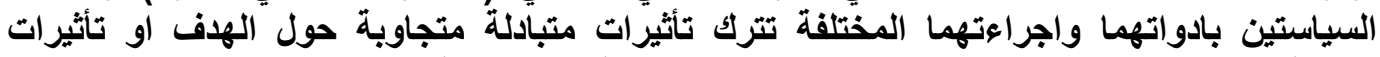

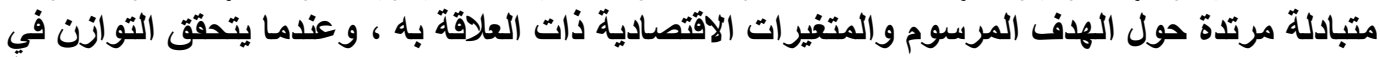

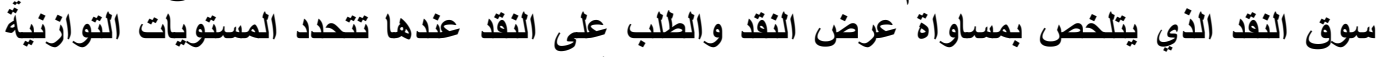

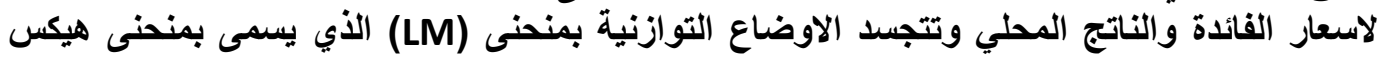

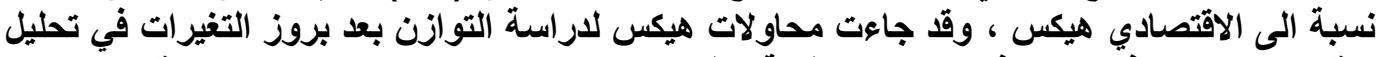

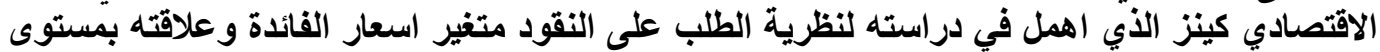

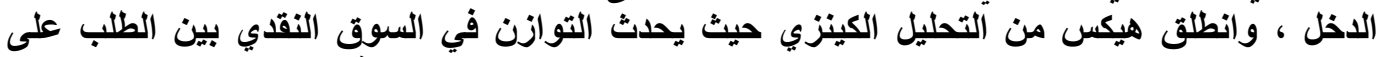

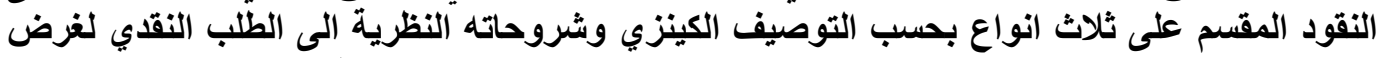

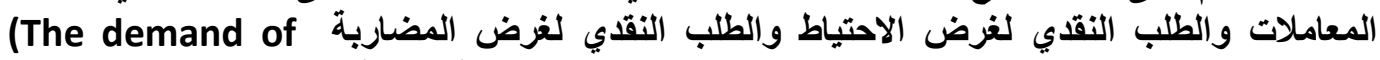
money for speculitve)

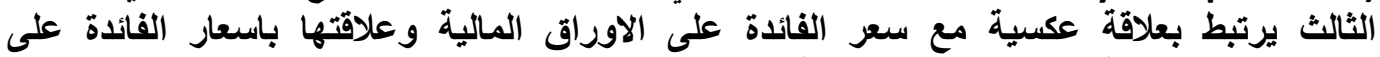
الاستثمارات الحقيقية بفعل القوى التصاحبية لاسعار الفائدة . 
ويمكن التعبير عن التوازن في السوق النقدي MD = MS بالمعادلات الاتية :-

MS = MD =MD1 + MD2

= الطلب النقدي لاغراض المعاملات والاحتياط ويكون دالة بالدخل . MD1

حيث ان

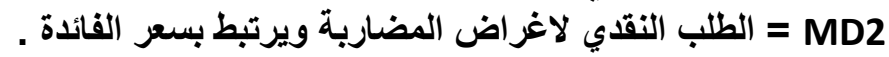

العرض النقدي المثبت من قبل السلطة النقدية كمتفير خارجي في دوال توازن السوق النقدي . MS MD1 = K (Y)

$M D 2=F(r)$

$M D 2=10-I$ ri

$M S=M D$

$M S=K Y+10-I r i$

$\mathrm{MS}-\mathrm{lo}+\mathrm{Iri}=\mathrm{K} Y$

$\mathrm{Y}=\mathrm{Ms}-\mathrm{lo}+\mathrm{Iri} / \mathrm{k}$

(د.احمد عبد الرحيم زردق ، التحليل الاقصادي الكلي، توازن الاقتصاد القومي ، كلية التجارة - جامعة

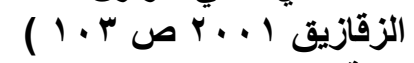

lo

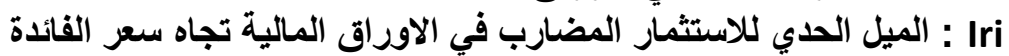

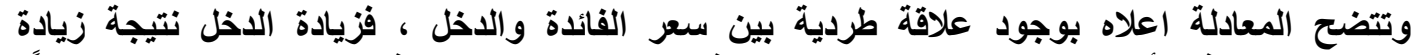
الاستثمارات تدفع بأتجاه زيادة الطلب على النقود بدافع المعاملات والاحتياط فتزداد واد استعار الفائدة تدريجياً. شكل (1) التوازن في السوق النقدي

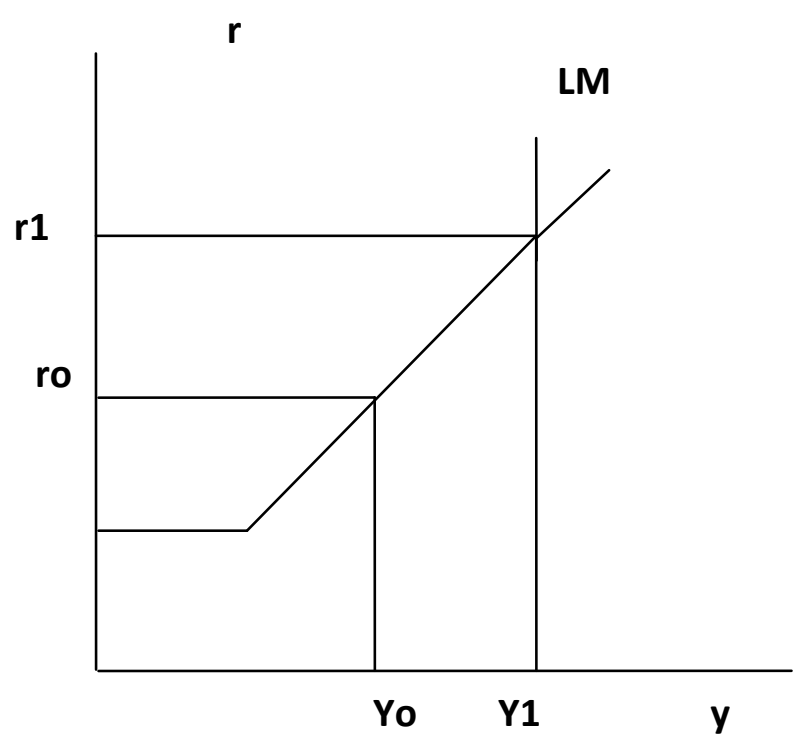

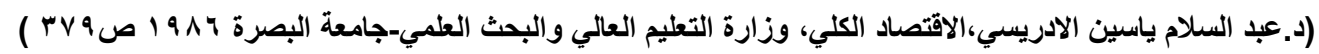


وفي حالة زيادة العرض النقدي على الطلب النقاي يقود الامر الى انخفاض اسعاد التعار الفائدة وزيادة

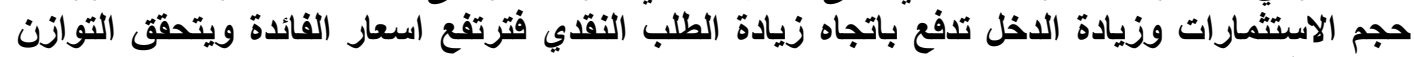

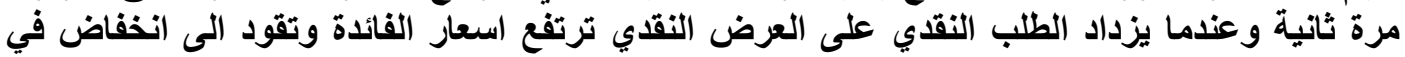

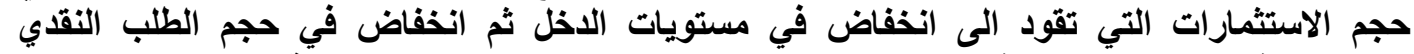

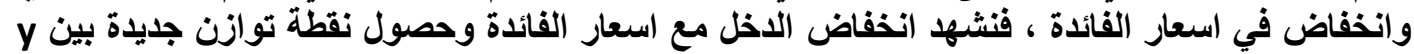
و r على منحنى LM و هذا ما يدلل على العلاقة الطردية بين الاخل الحقيقي وسعر الفائدة .

$M D=F(Y, r)$

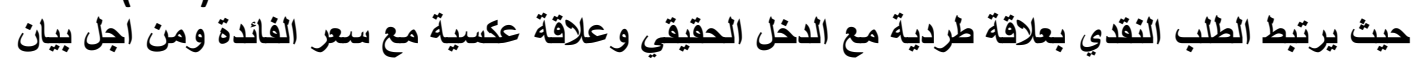

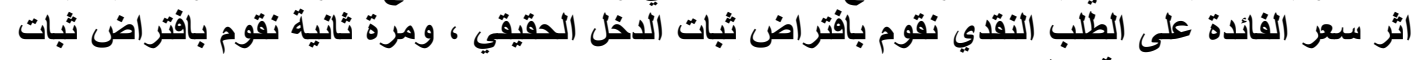

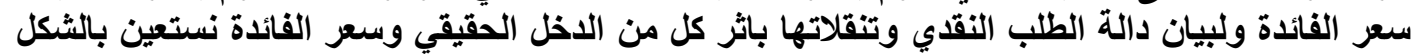

( ادناه : (7)

شكل رقم (2) علاقة الطلب النقدي بسعر الفائدة

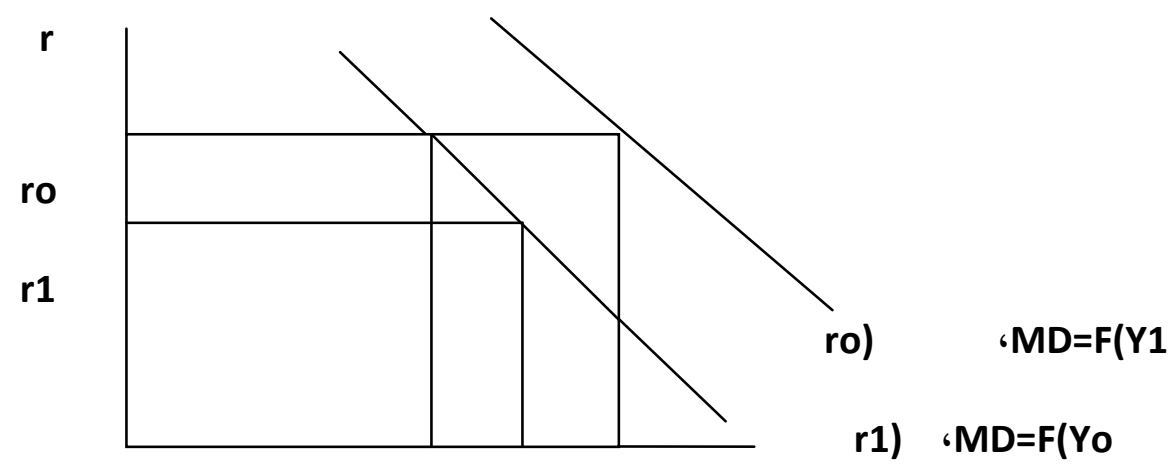

Mdo Md1 Md2 MD

(مايكل ابلجمان ـ الاقتصاد الكلي ، النظرية والسياسة ، تعريب محمد ابراهيم منصور ، دار المريخ للنشر ، السعودية ، 999 ، 19 ، ؛ ( $r \leq \leqslant \leq$ 


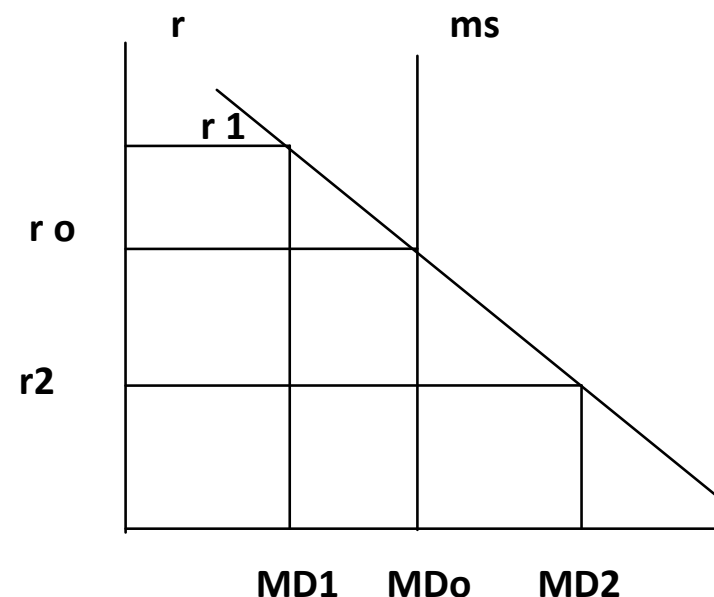

(مايكل ابدجمان - الاقتصاد الكلي ، النظرية والسياسة ، تعريب محمد ابراهيم منصور ، دار المريخ للنشر ، السعودية ،

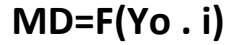

$\mathrm{MD}=\mathrm{MS}$

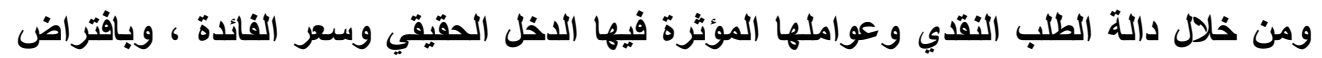

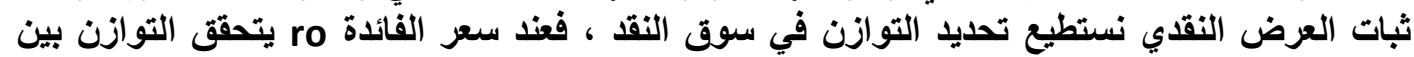

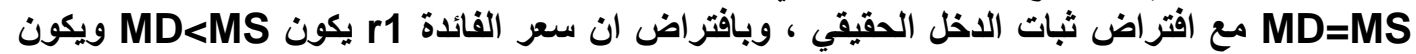

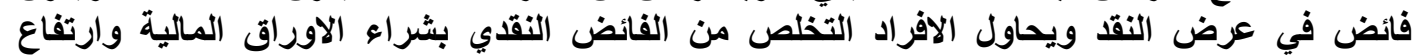

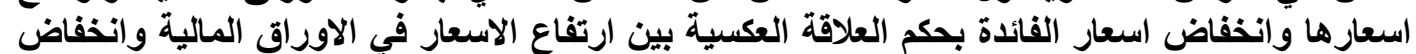

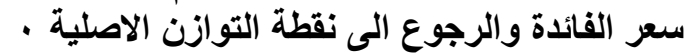

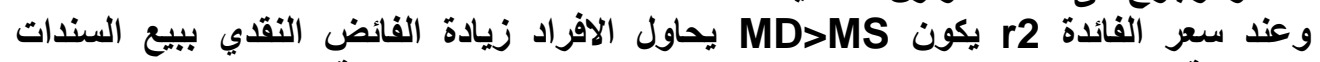

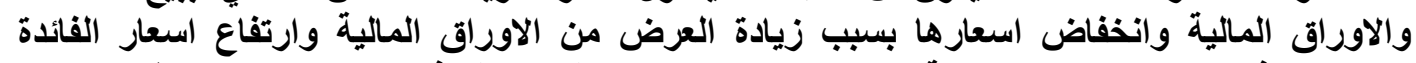

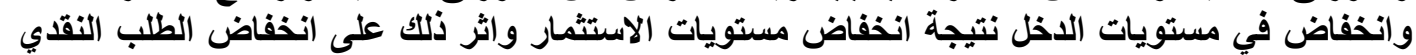

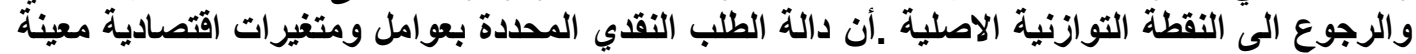

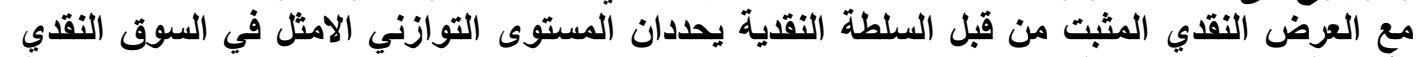

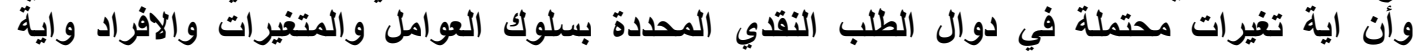

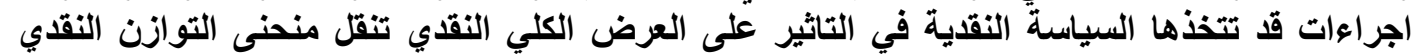

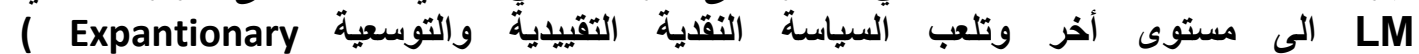
\&contractionary policy)

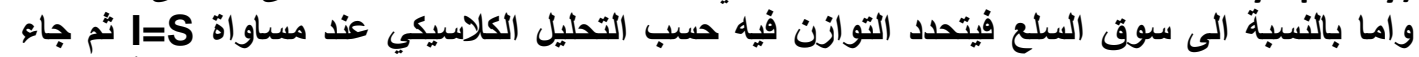

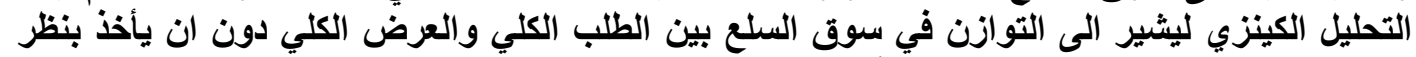

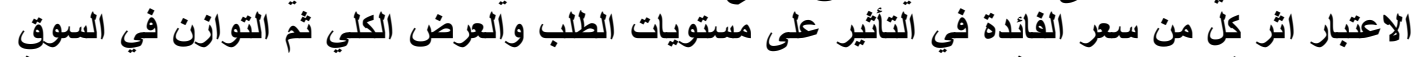

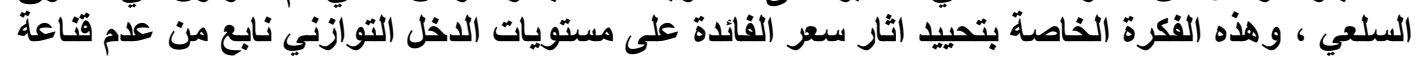

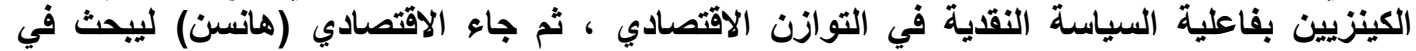

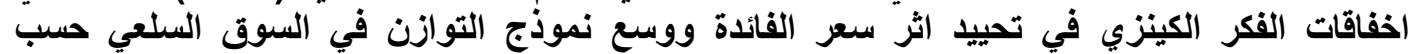
المنظور الكلاسيكي . اكنزي 


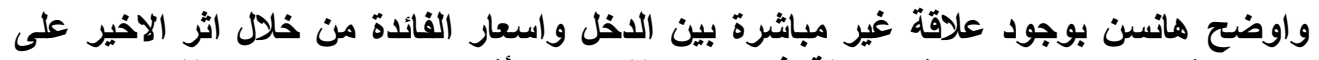

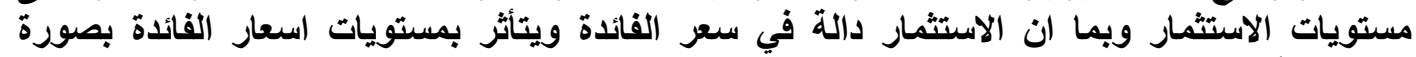

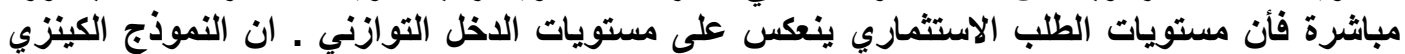

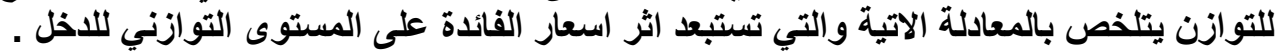

$\mathrm{y}=\mathrm{C}+\mathrm{lo}+\mathrm{Go}+\mathrm{Xo}-\mathrm{M}$

$y=a+b y+l o+X o-(M o+m y)$

$\mathrm{y}=\mathrm{a}+\mathrm{by}+\mathrm{lo}+\mathrm{Go}+\mathrm{Xo}-\mathrm{Mo}-\mathrm{my}$

$y-b y+m y=a+l o+G o+X o-M o$

$y(1-b+m)=a+10+G o+X o-M o$

$\mathbf{y}=\frac{\mathrm{a}+\mathrm{by}+\mathrm{Io}+\mathrm{Go}+\mathrm{Xo}-\mathrm{Mo}}{1-\mathrm{b}+\mathrm{m}}$

امـا النموذج الذي طوره الاقتصادي هانسن بإدخال اثر سعر الفائدة على المستوى التوازني للاخل

$\mathrm{y}=\mathrm{C}+\mathrm{I}+\mathrm{Go}+\mathrm{Xo}-\mathrm{M}$

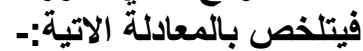

$\mathrm{C}=\mathrm{a}+\mathrm{by} \quad \mathrm{I}=\mathrm{lo}-\mathrm{kr} \quad \mathrm{M}=\mathrm{Mo}+\mathrm{my}$

$\mathrm{y}=\mathrm{a}+\mathrm{by}+\mathrm{lo}-\mathrm{kr}+\mathrm{Go}+\mathrm{Xo}-\mathrm{Mo}-\mathrm{my}$

$y-b y+m y=a+l o-k r+G o+X o-M o$

$y(1-b+m)=a+l o-k r+G o+X o-M o$

$\mathbf{y}=\frac{\mathbf{a}+\mathbf{I o}-\mathbf{k r}+\mathbf{G o}+\text { Xo-Moy }}{\mathbf{1}-\mathbf{b}+\mathbf{m}}$

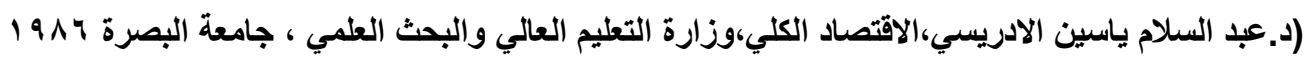

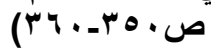

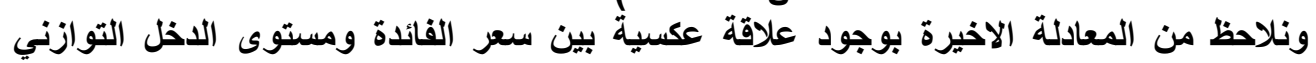

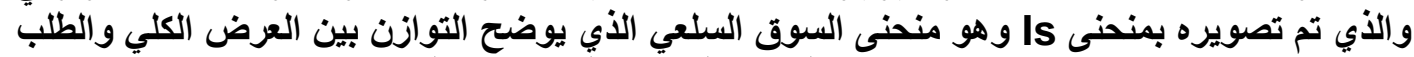

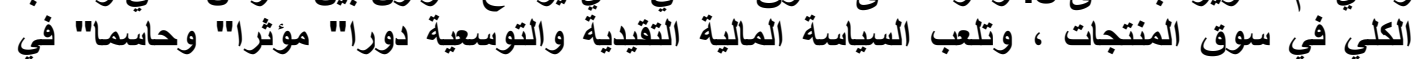

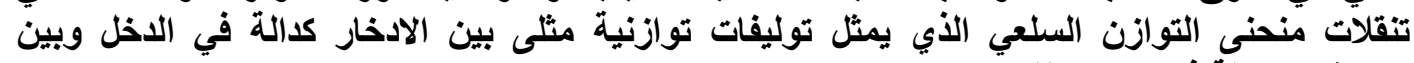

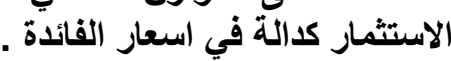

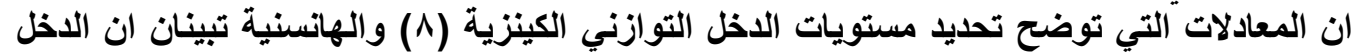

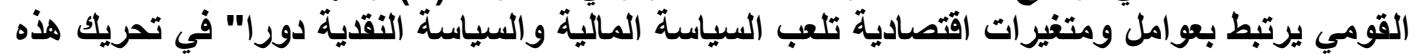

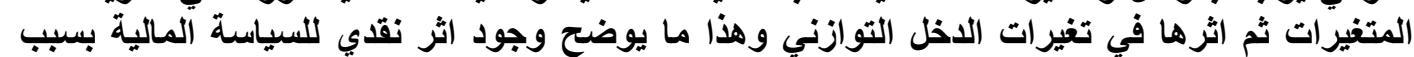

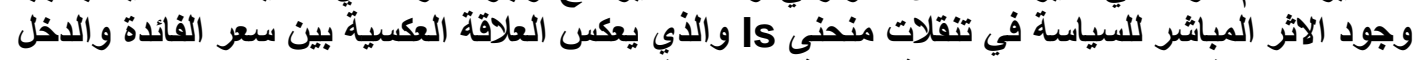

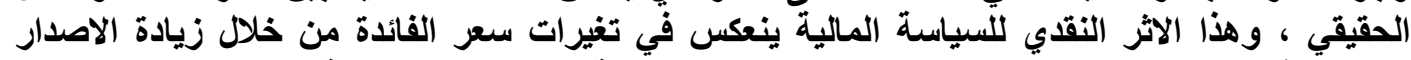

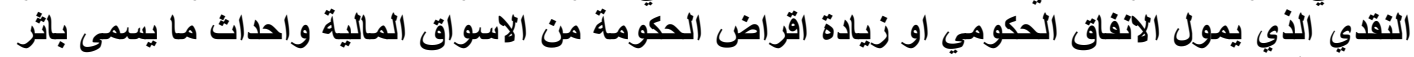

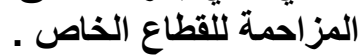


التوازز الاقتنصادي العام ( IS-LM )

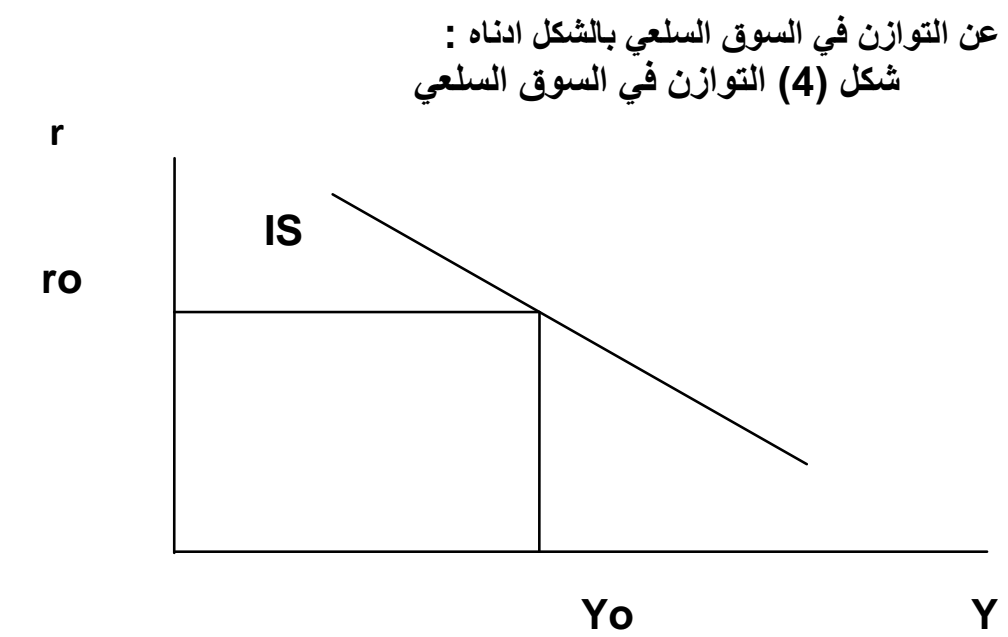

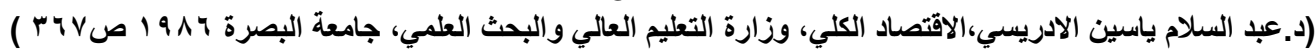

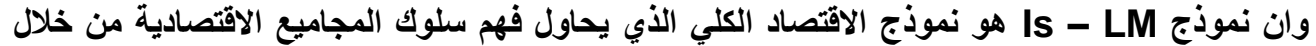

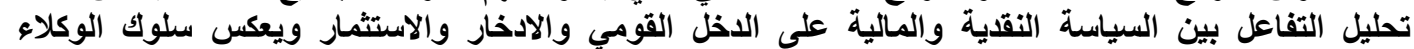

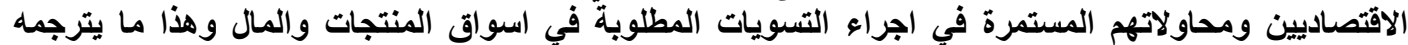

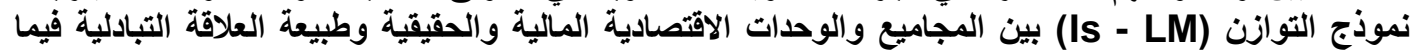

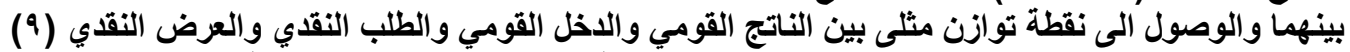

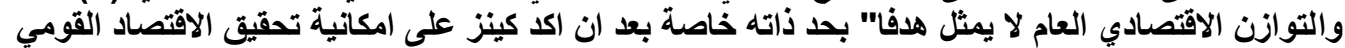

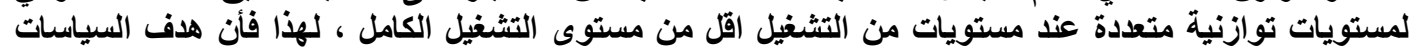
الاقتصادية ينصب على تحقيق الاستقرار الاقتصادي والنقدي اكثر من من الاهتمام بتحقيق التوازن الاقتصادي التئي

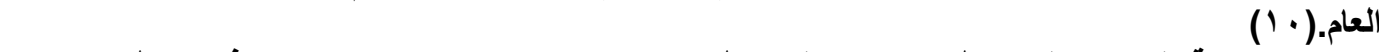
ان صيغة التوازن العام تظهر من خلال تقاطع منحنى (IS) مع منحنى (LM) وفي تقاطعهما يتحدد المستوى التوازني للاخل وسعر الفائدة .

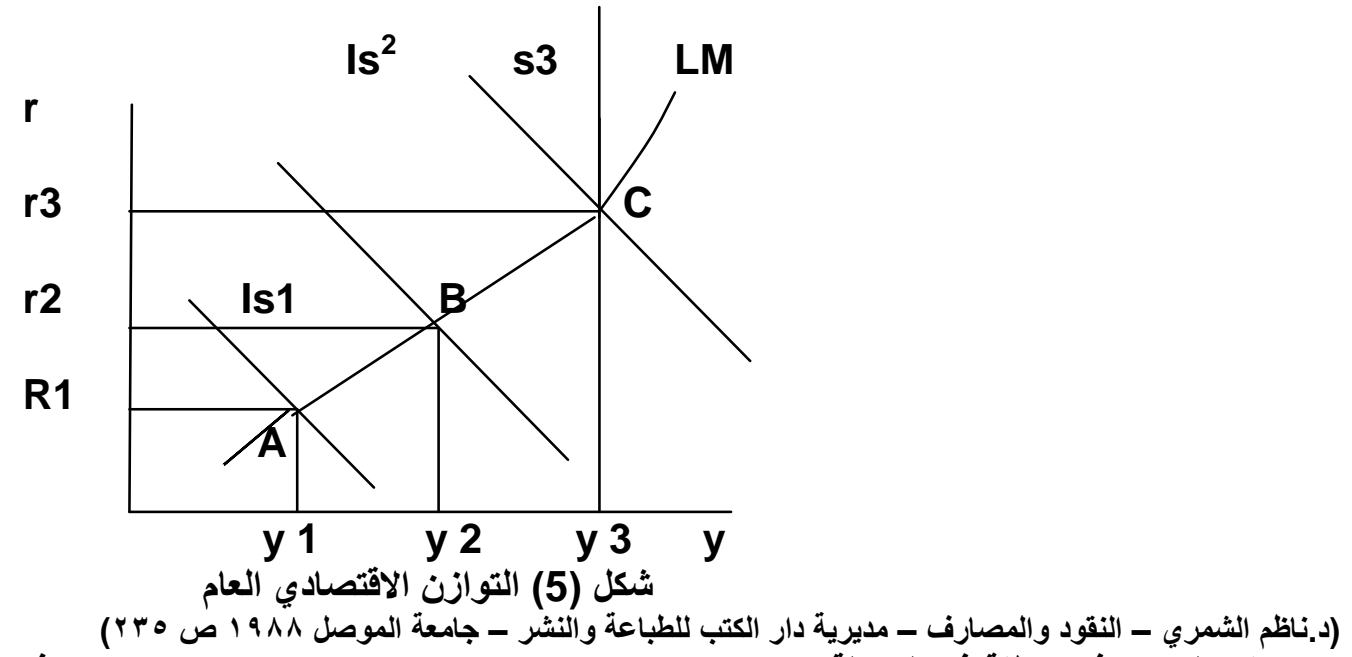

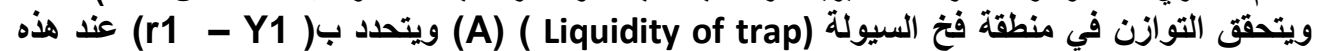

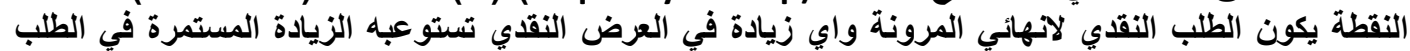

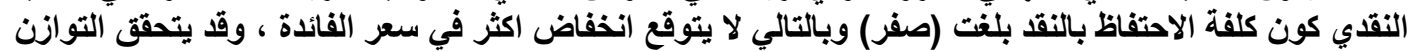

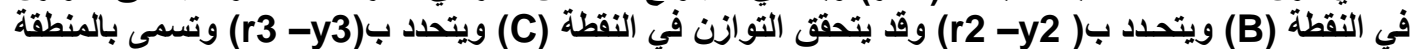

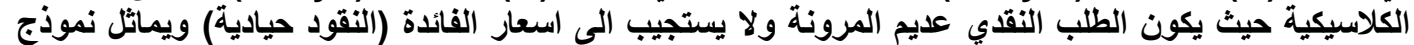
التوازن العام نموذج الطلب الكلي والعرض الكلي الكينزي واثر زيادات الطلب النقائي على الاسعار حتى بلوغ

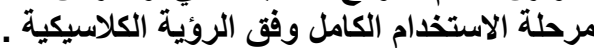




\section{المبحث الثاني /التوازن الاقتصادي المستقر وغير المستقر}

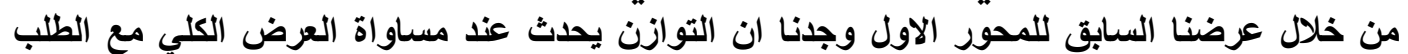

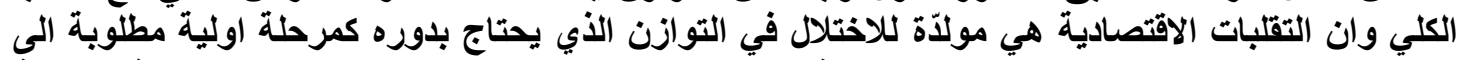

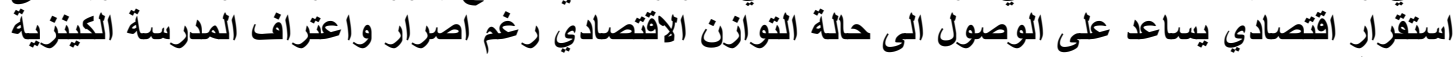

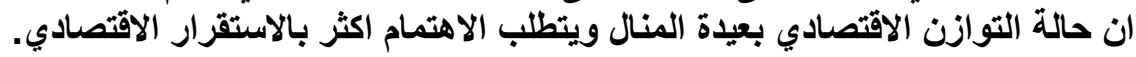
ان التوازن الاقتصادي المستقر (The stablize economic balance) هو حالة قابلة اللى اعادة

The التوازن في حالة تعرض هذا التوازن الى اختلال بفعل قوى ذاتية وان التوازن الاقتصادي غير المستقرة non- stablize economic balance

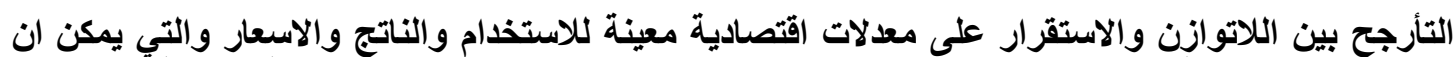

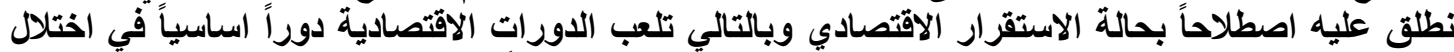

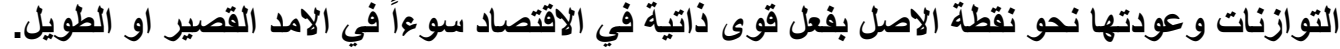

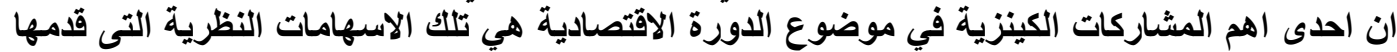

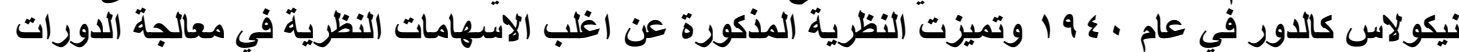

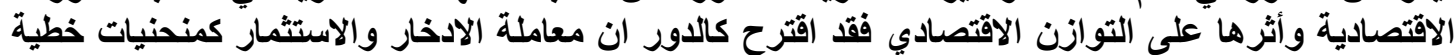

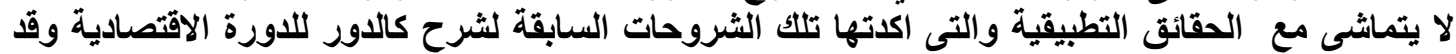

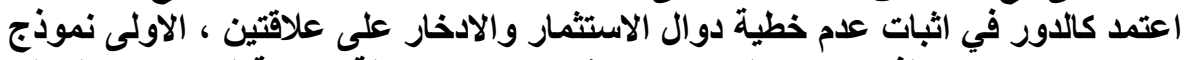

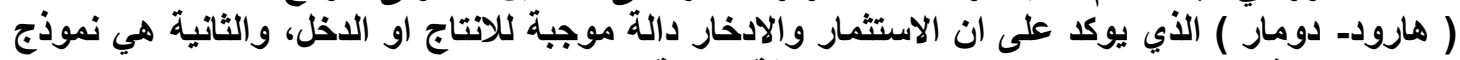

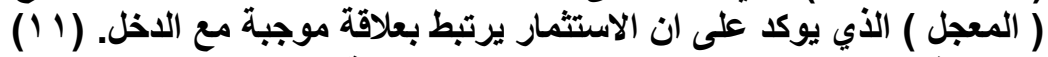

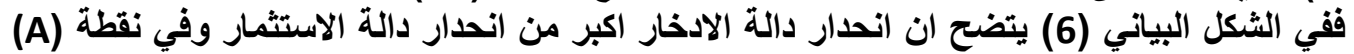

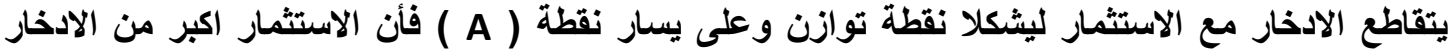

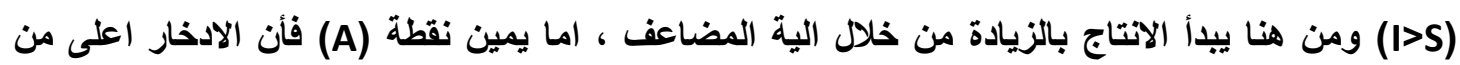

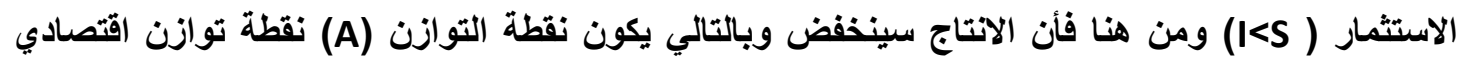

$$
\text { الثكل (7) التوازن الاقتصادي غير المستقر الثكل (6) التوازن الاقتصادي المستقر }
$$

S.I

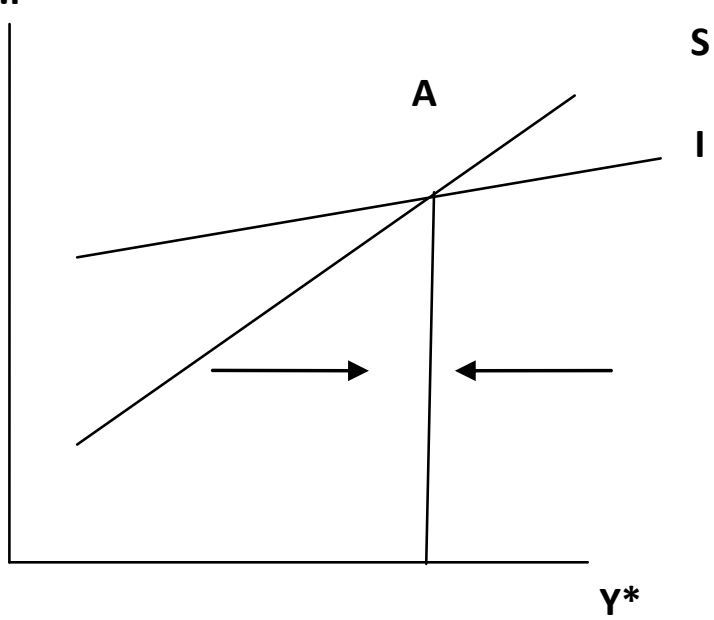

S.I

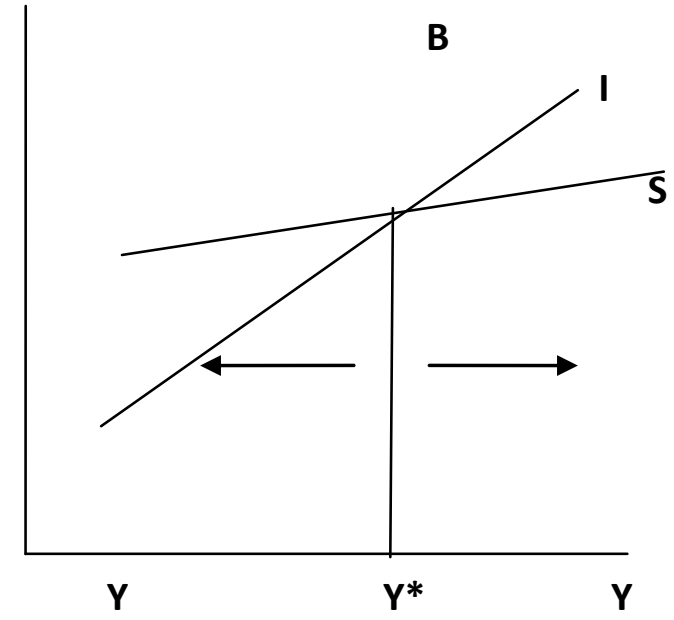

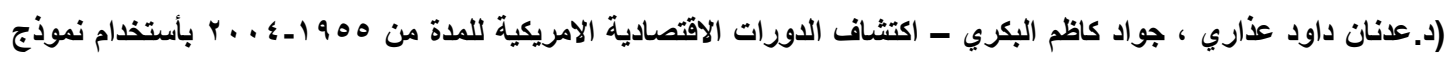

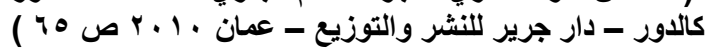




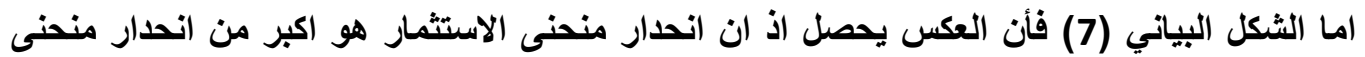

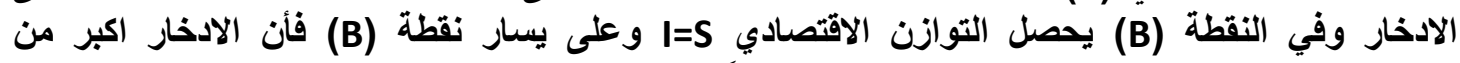

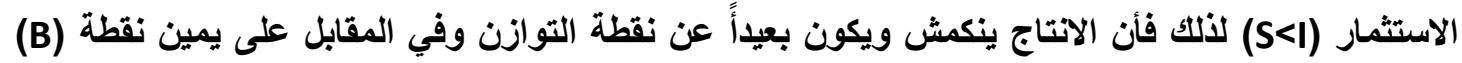

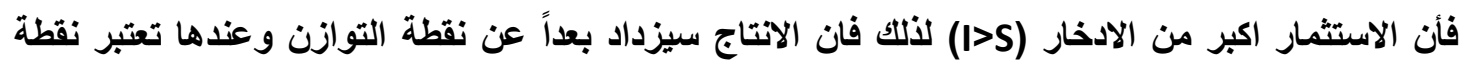

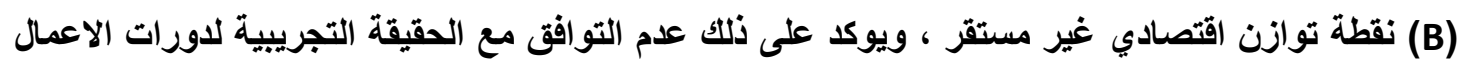

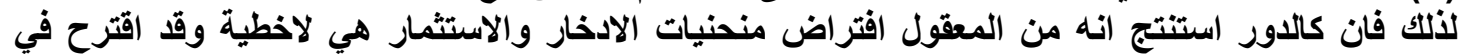

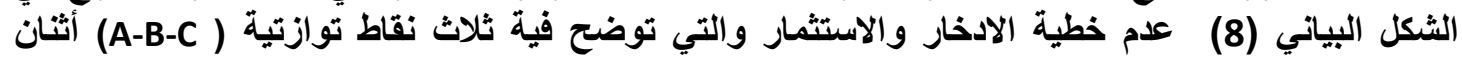

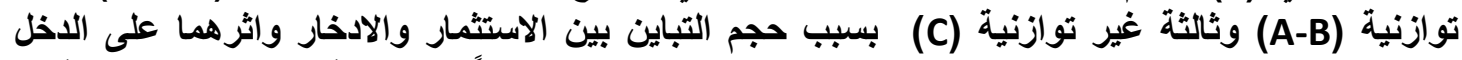

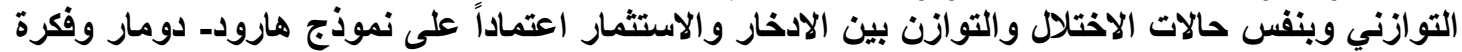
المضاعف و المعجل. الات

شكل (8) التوازن الاقتصادي المستقر وغير المستقر والاورات الاقتصادية

\section{S.I}

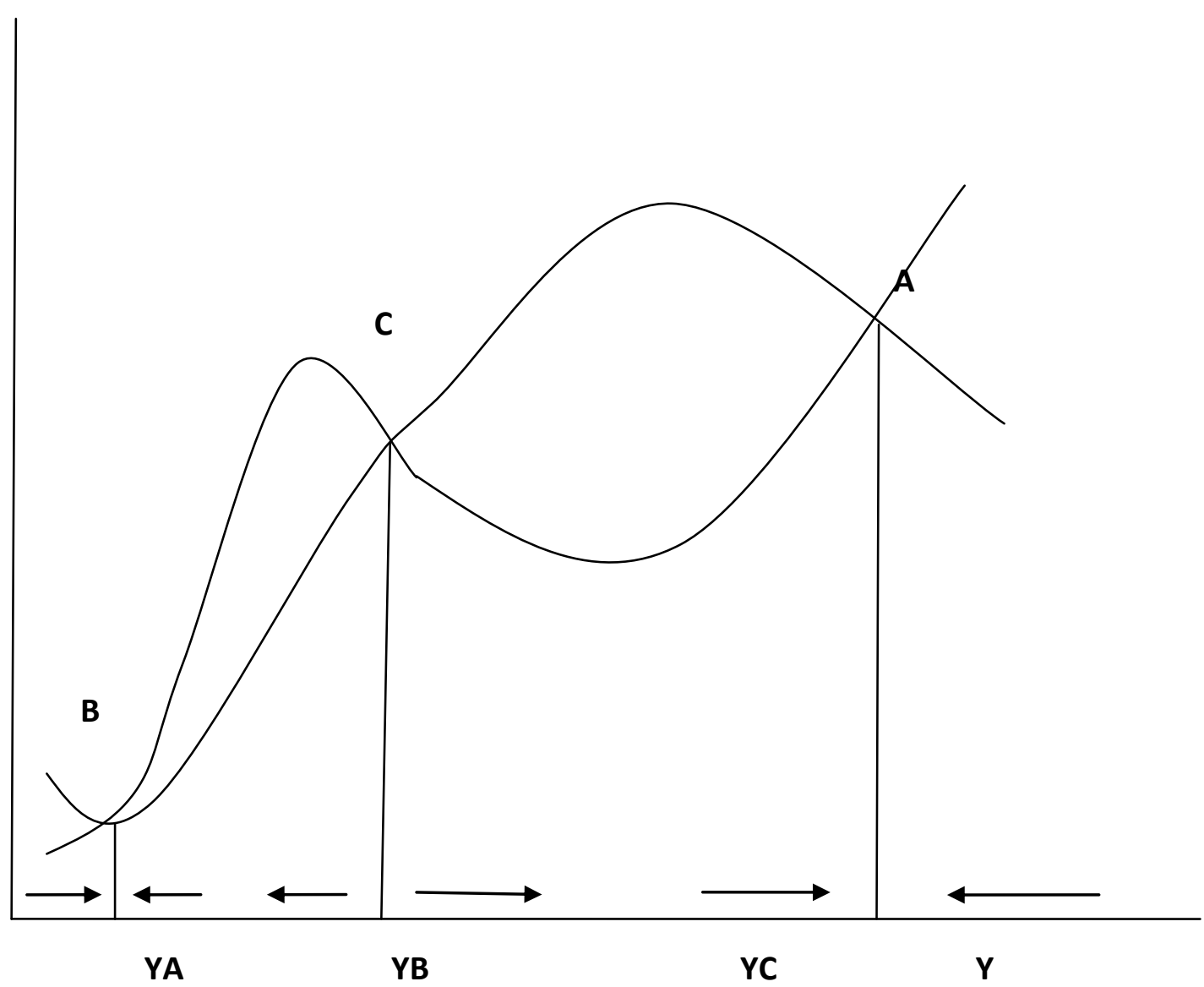

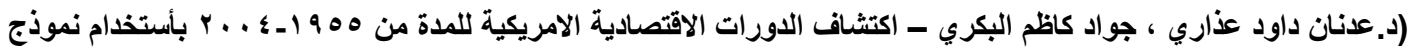

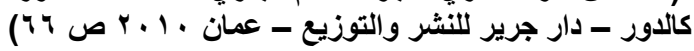

لذا صفوة ما تقدم ان الدورات الاقتصادية نتاج تقاطع في نقاط توازنية غير مستقرة وان التان التوازن

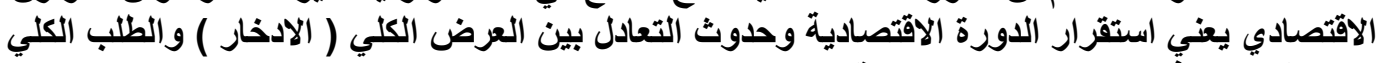

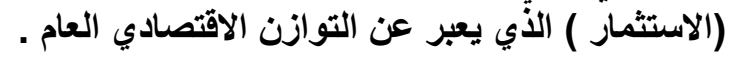




\section{المبحث الثالث/التأثيرات المتبادلة للسياسة النقدية والمالية على التوازن}

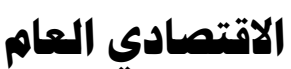

بعد عرض التوازن الاقتصادي العام الذي ينعكس في التوازن النقدي والتوازن السلعي وكيف ان الني التئي

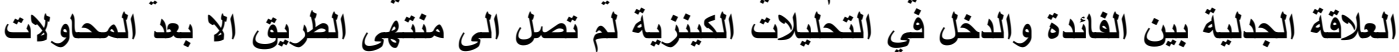

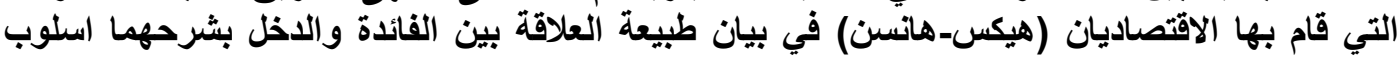

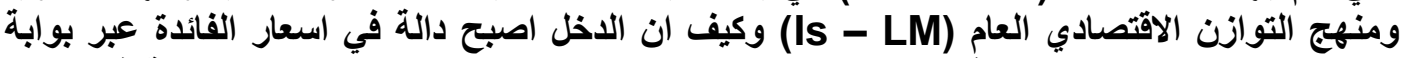

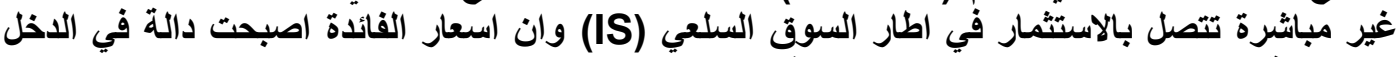

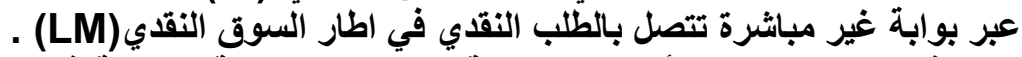

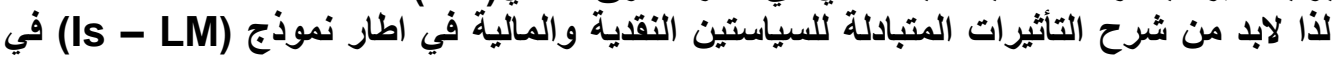

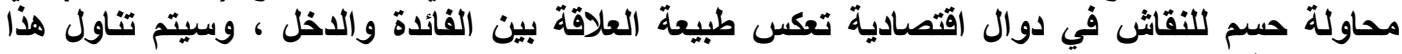

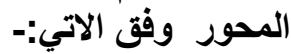

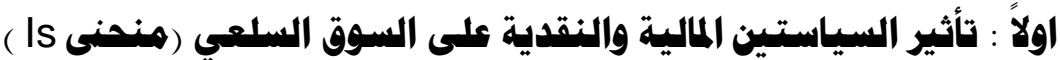

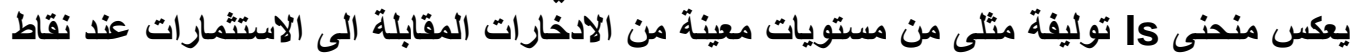

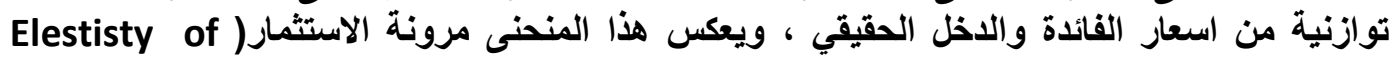
(investment

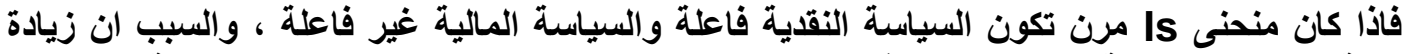

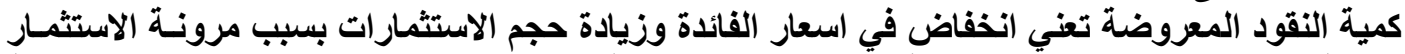

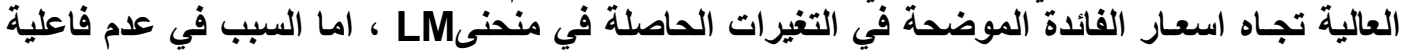

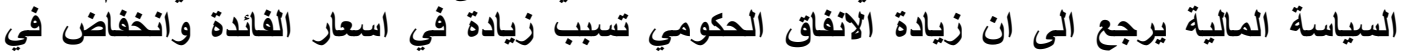

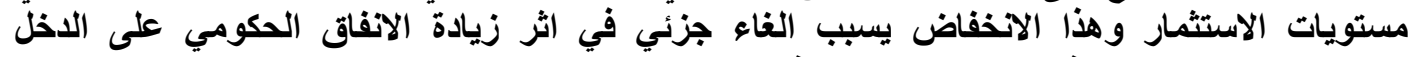

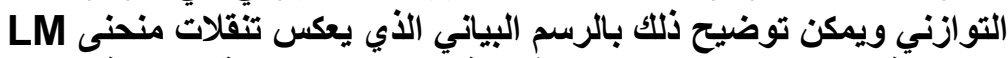

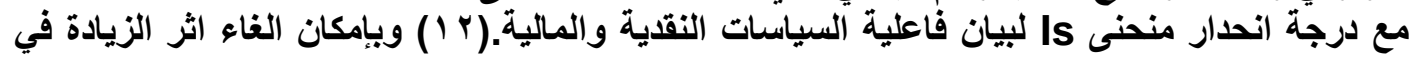

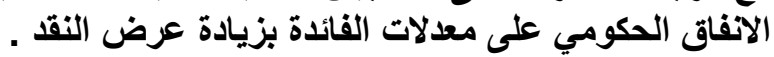

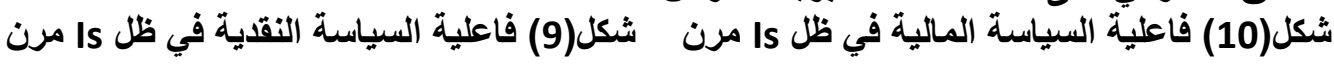
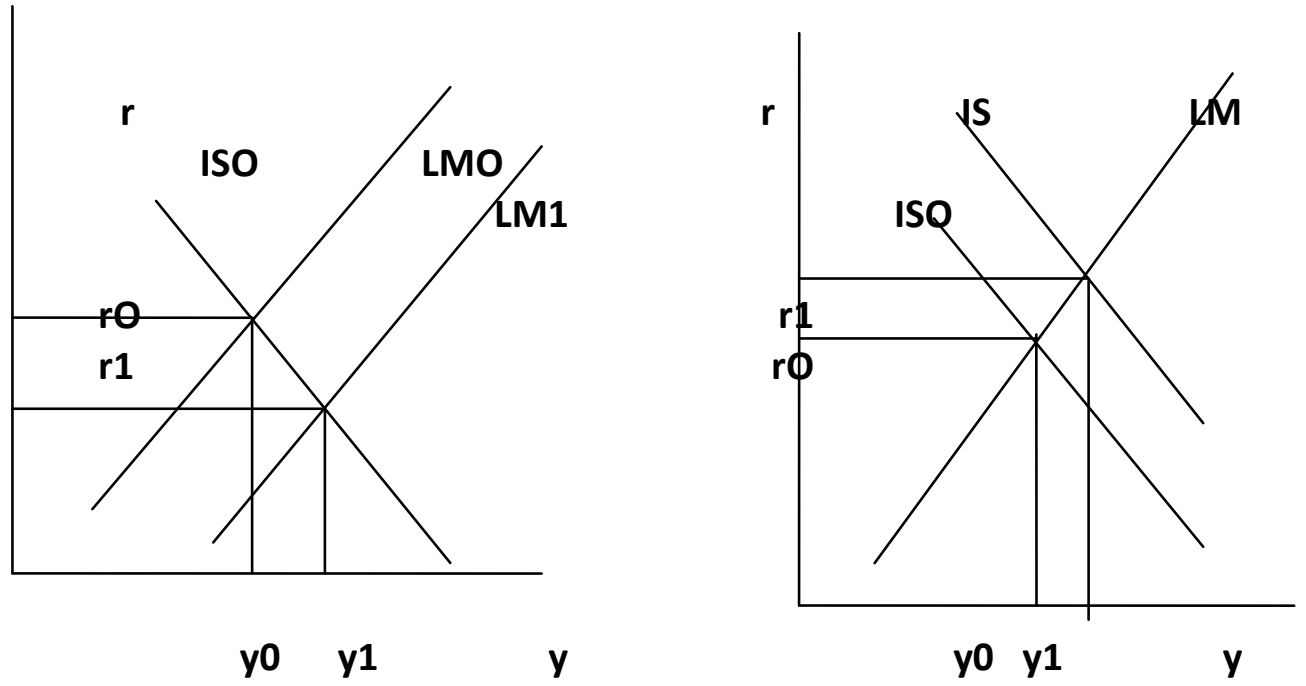

(د.سامي خليل ، نظرية الاقتصاد الكلي ـالمفاهيم والنظريات الاساسية _الكتاب الاول ـ الكويت ؛ 99 ص .9 ؛ ) 
اما اذا كان منحنى Is غير مرن والأي يعكس انخفاض مرونة الاستثمار تجاه اسعار الفائدة فان إنان

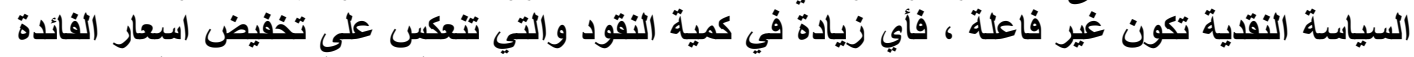

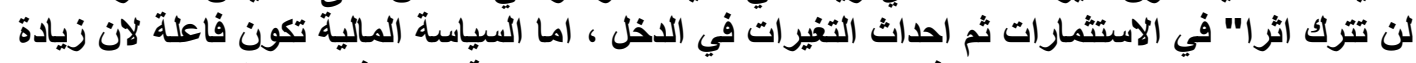

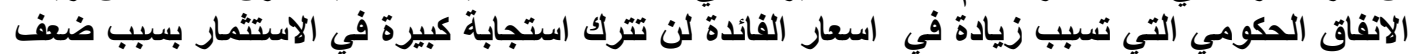

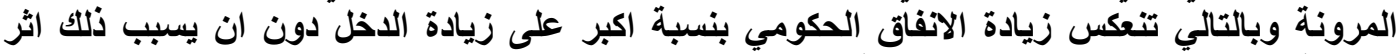

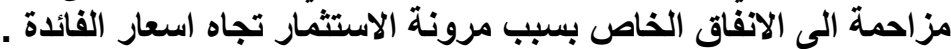

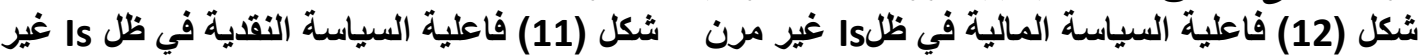
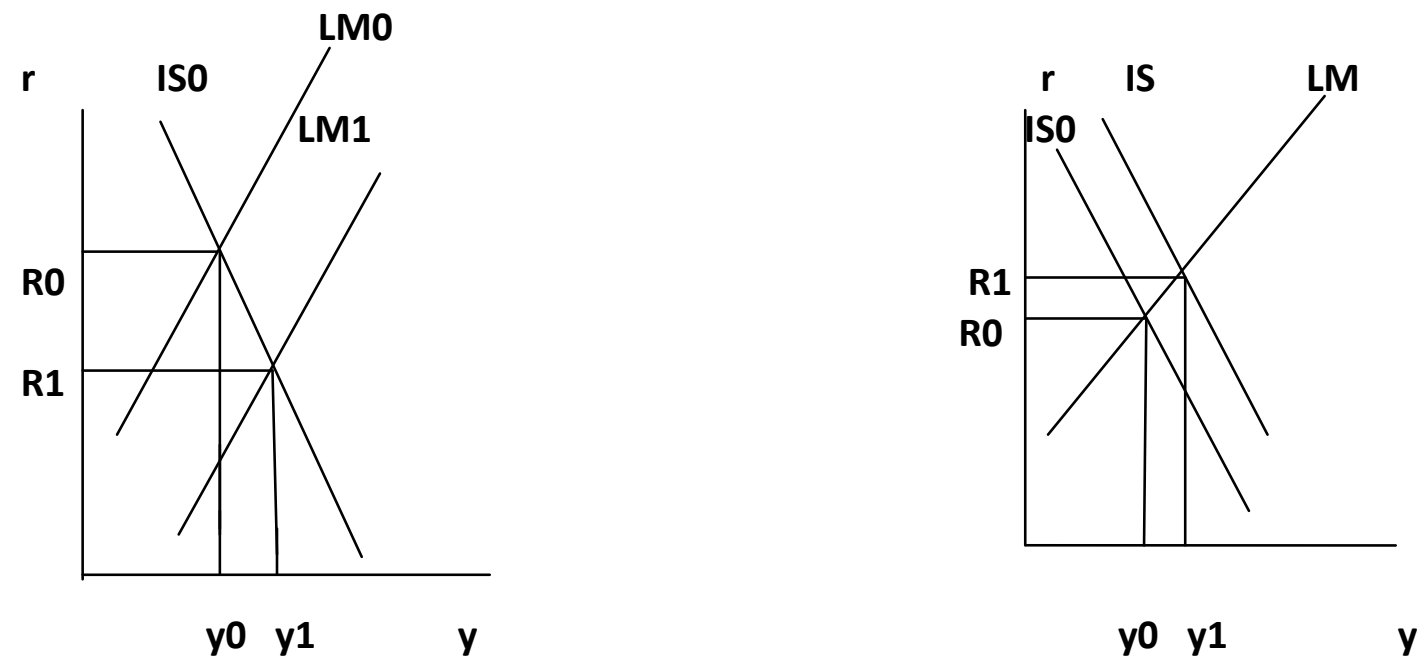

(د.سامي خليل ، نظرية الاقتصاد الكلي ـالمفاهيم والنظريات الاساسية ـالكتاب الاول ـ الكويت ؛ 9 1 ـ ص ب ج ؛)

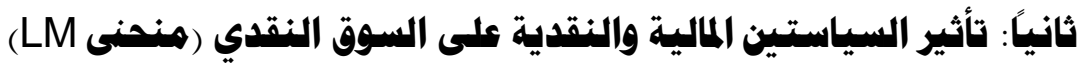

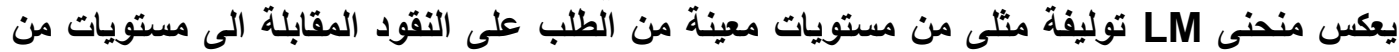

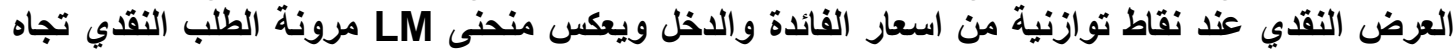

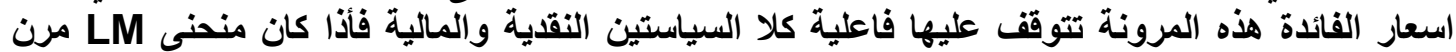

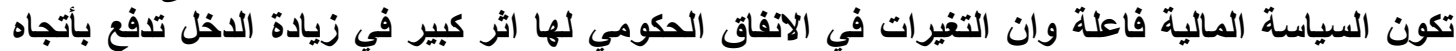

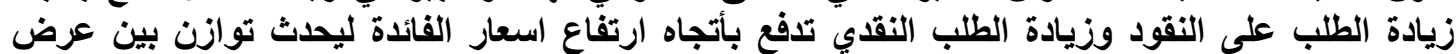

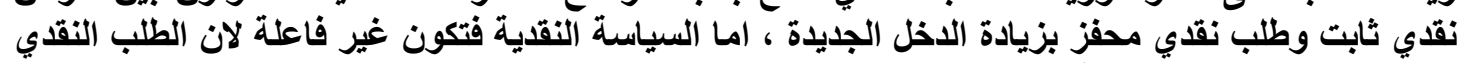

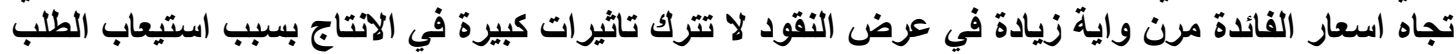

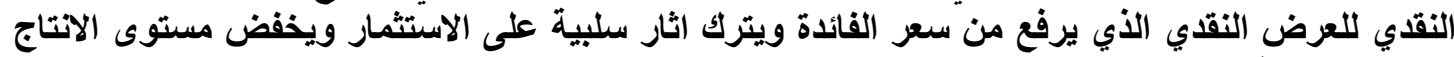
كما موضحان في الثكل الآتي : 
شكل (14) فاعلية السياسة النقدية في ظل LM مرن شكل(13) فاعلية السياسة المالية في ظل LM مرن
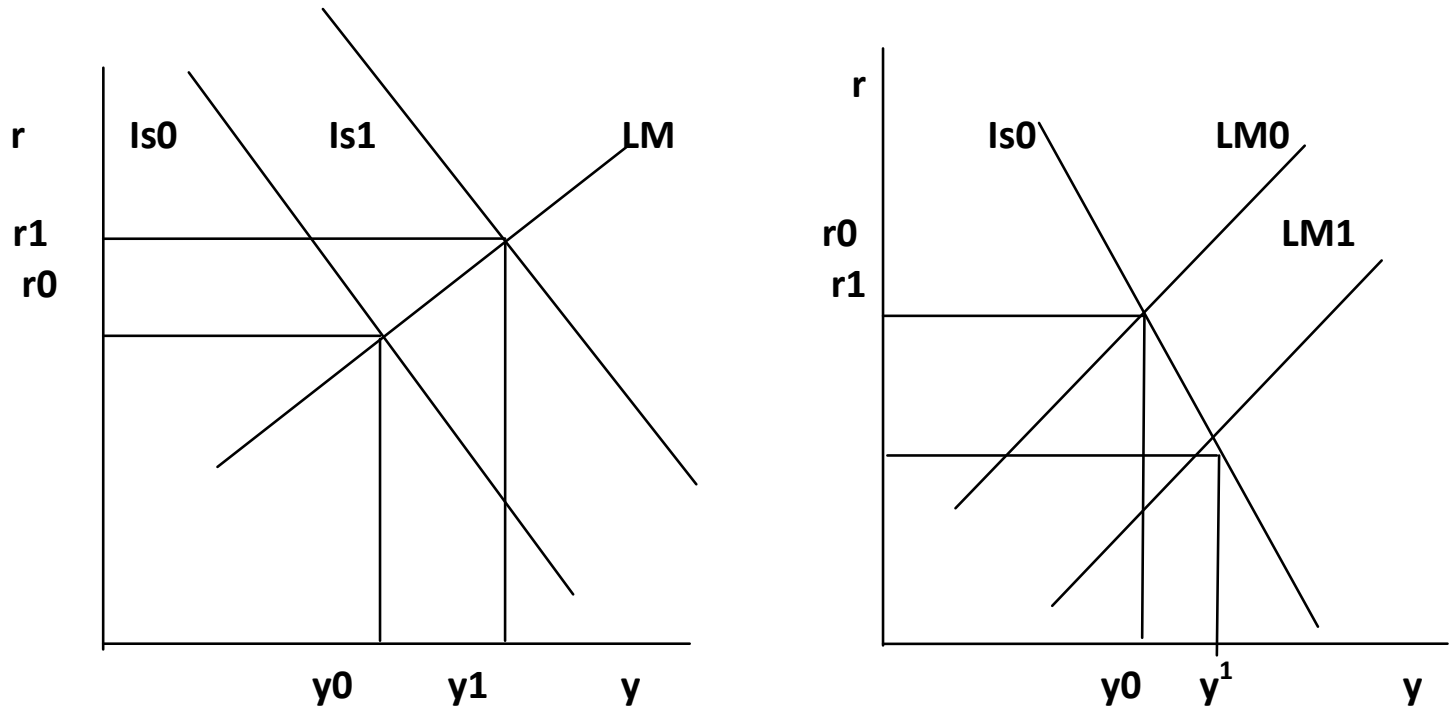

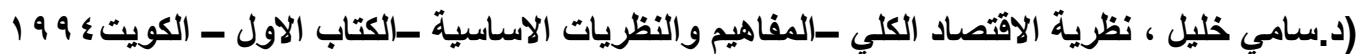

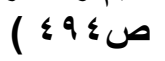

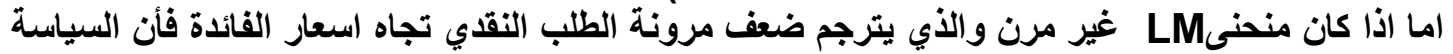

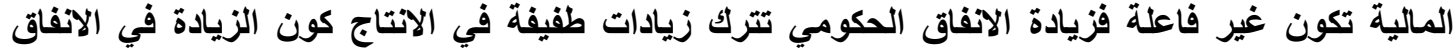

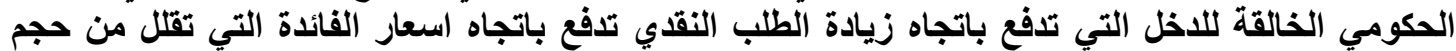

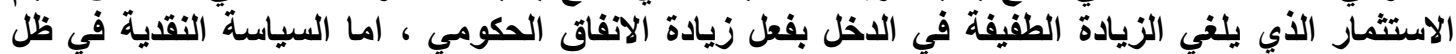

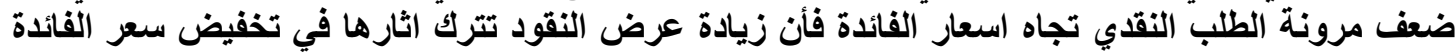

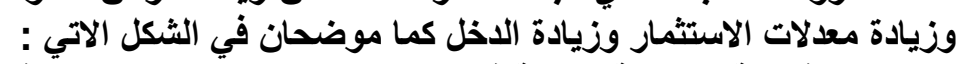

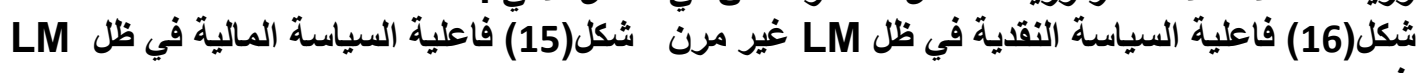

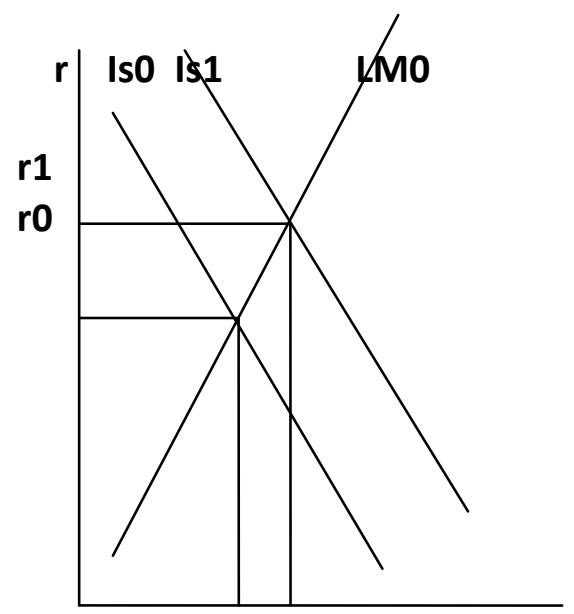

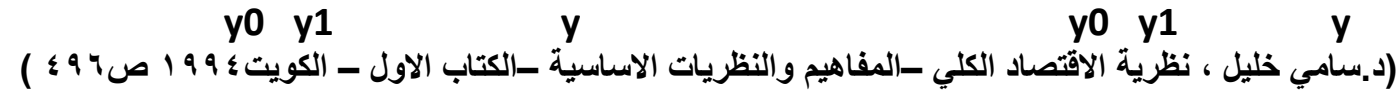

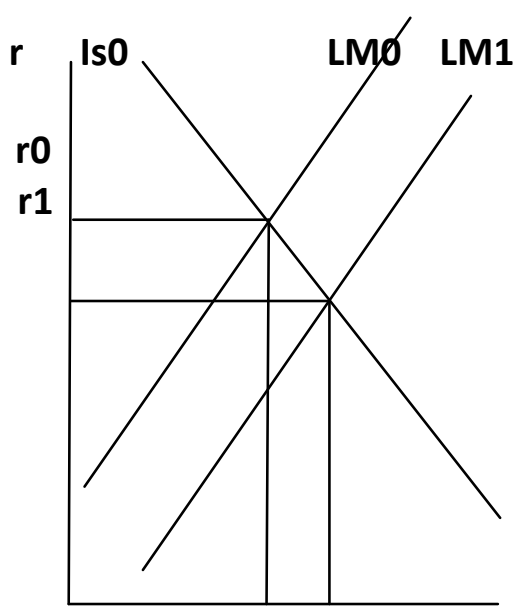

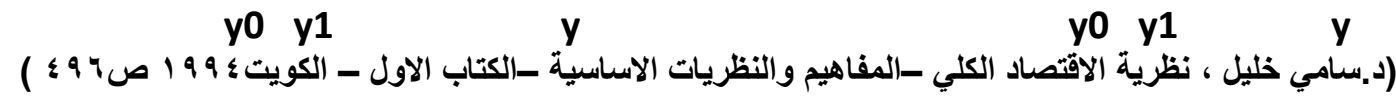
غير مرن 
اذن صفوة القول : تتوقف فاعلية السياستين المالية والنقدية على مرونة الاستثمار تجاه اسعار الفائدة

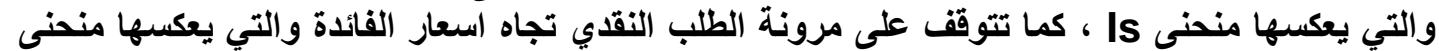

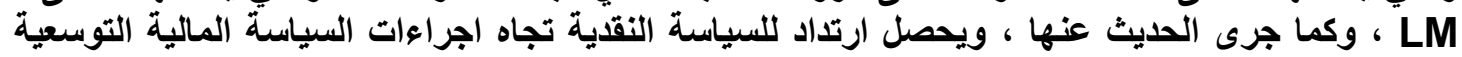

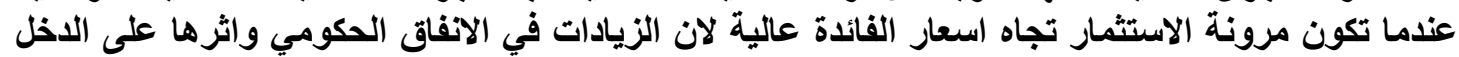

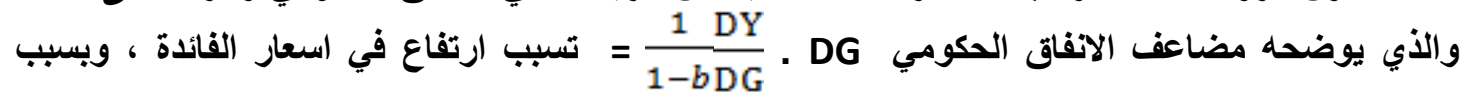

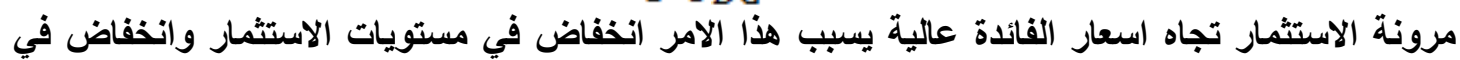

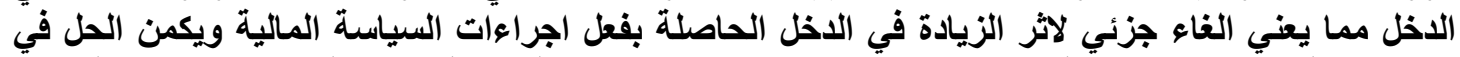

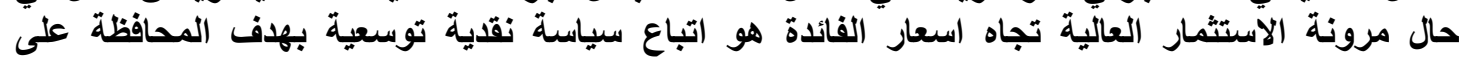

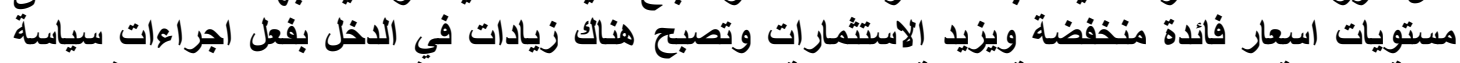

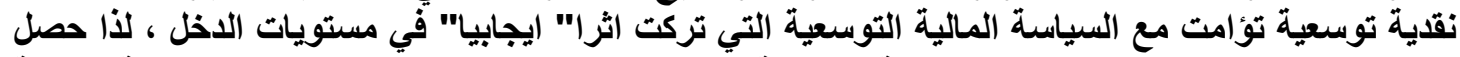

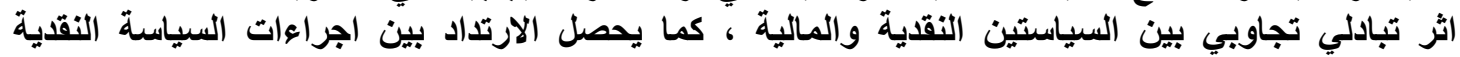

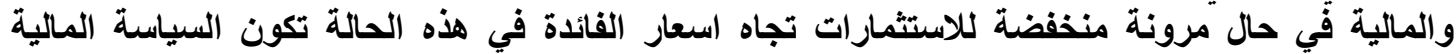

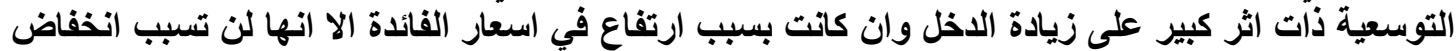

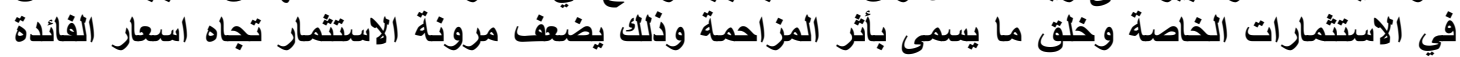

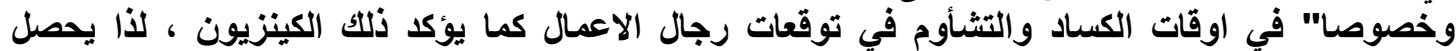

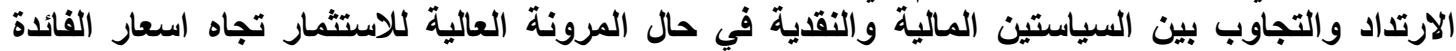
ويحصل فقط الارتداد للسياسة النقاية في حال المرونة المنففضة للاستثمار تجاه استعار الفئة الفائدة وبالتالي تكون

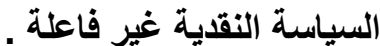

وكذلك تتوقف فاعلية السياستين النقدية والمالية على المرونة العالية للطلب النقاي تجاهية اسعار الفائدة

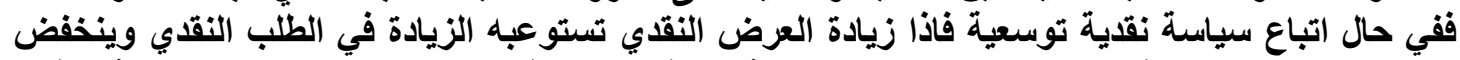

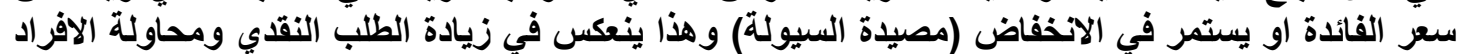

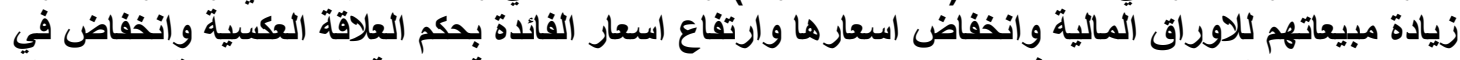

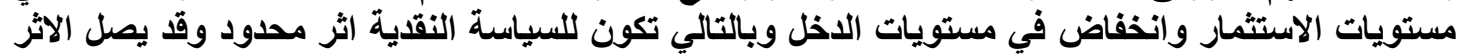

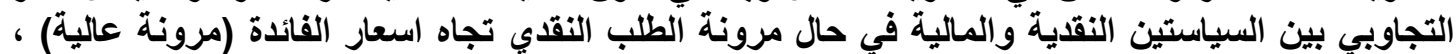

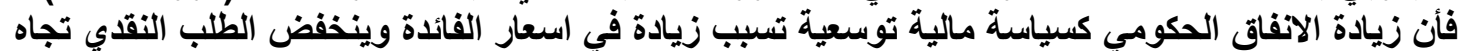

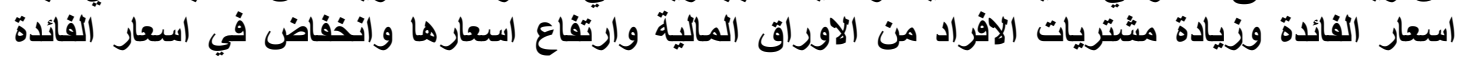

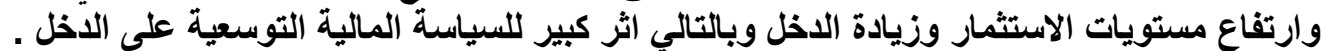

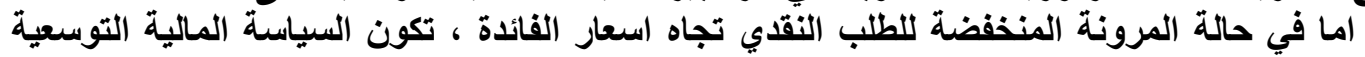

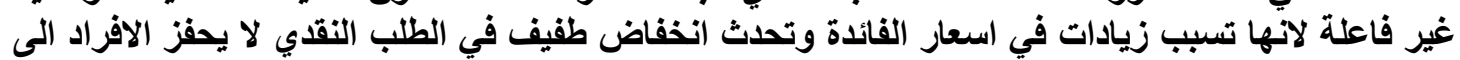

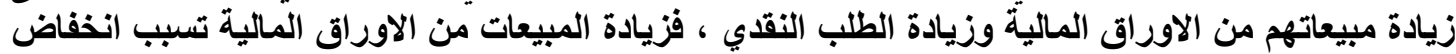

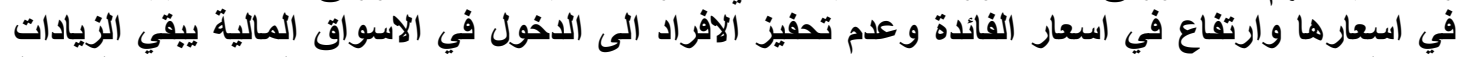

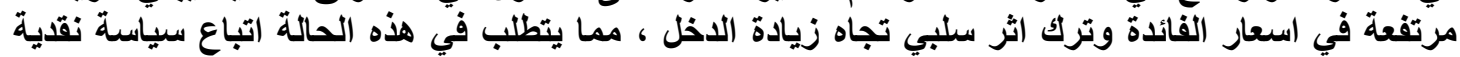

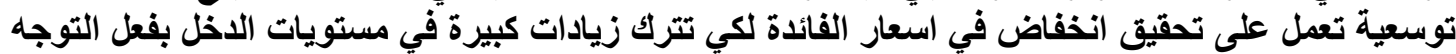

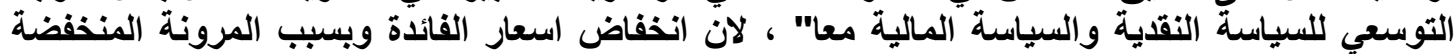

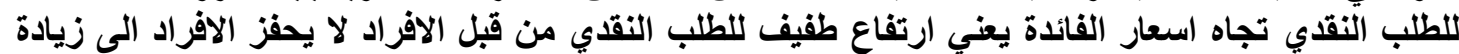

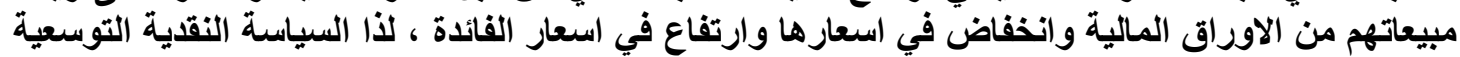

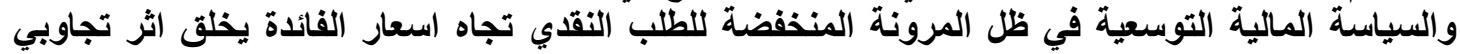
كبير بينهما تجاه حصول زيادات غير محدودة في في الدخل . 


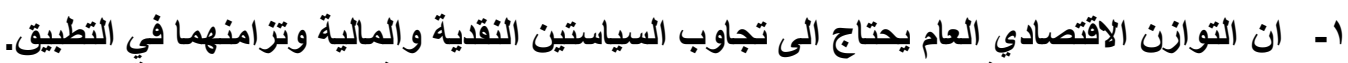

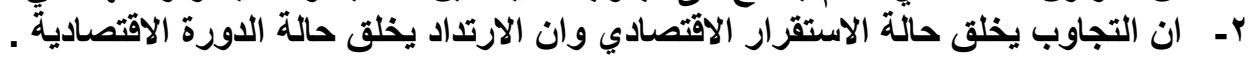

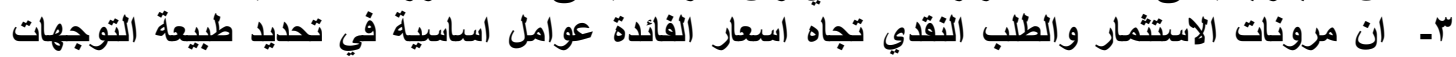

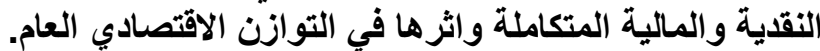

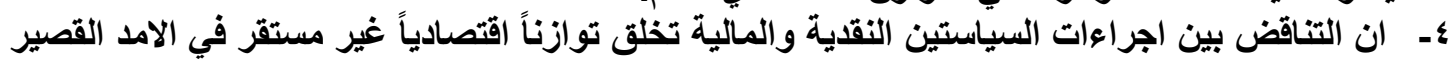

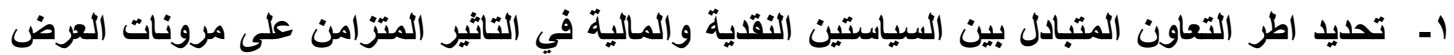

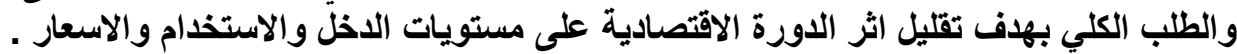

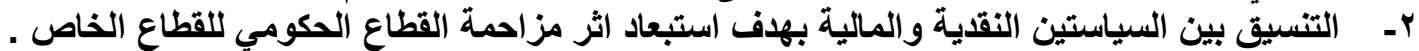

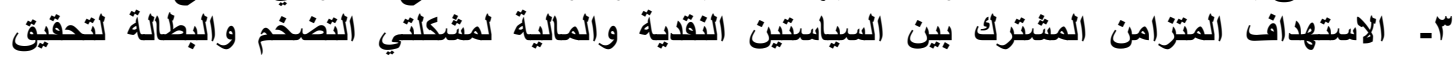
الاستقرار والنمو الاقتصادي.

1-Nicolas Kaldor "The Irrelevance of equilibrium Economic" The Economic 1972 .p:1،Journal . vol :82 .no:328

2-James R. Markusen "Introduction to Game for economic Equilibrium 2005 p.p 12-13 'problem" University of Colorado

وــ د.علي مجيد حمادي ـالتشابك الاقتصادي بين النظرية والتطبيق ـ دار اليازوري العلمية للنشر

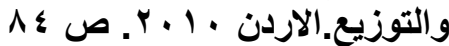

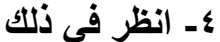

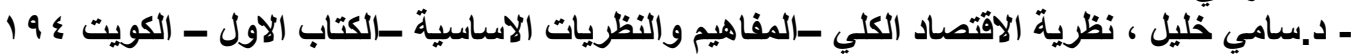

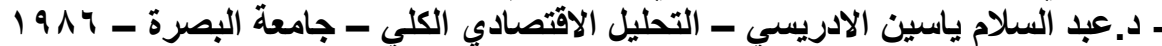

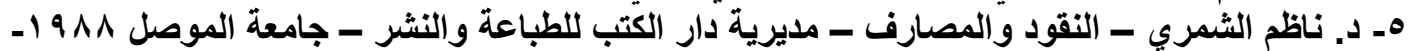

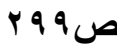
، المايكل ابلجمان - الاقتصاد الكلي ، النظرية والسياسة ، ، ، تعريب محمد ابراهيم منصور ، دار المريخ للنشر

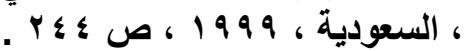

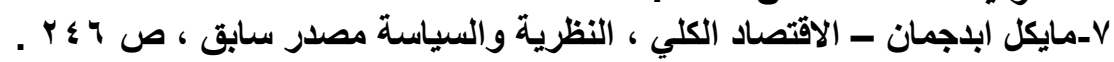

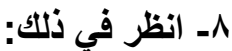

د..حمد عبد الرحيم زردق ، التحليل الكلي في قياس الاداء الاقتصادي ، كلية التجارة - جامعة الزقازيق . r...

دـ داحمد عبد الرحيم زردق ، التحليل الاقصادي الكلي، توازن الاقتصاد القومي ، كلية التجارة - جامعة

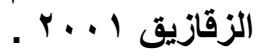

9 - Elmer G. Wiens "macroeconomics Theory \& Application" working paper 2011-p;1

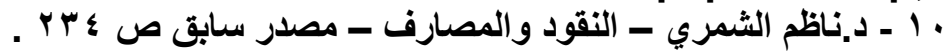

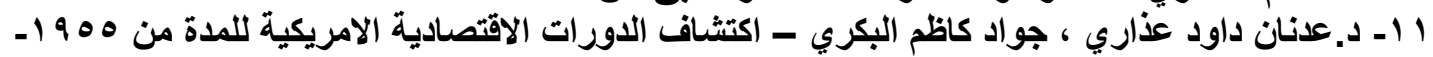

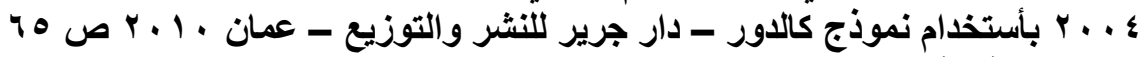

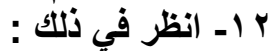
د.سامي خليل ـ نظرية الاقتصاد الكلي ـ الكتاب الاول ـ مصدر سابق ص • ـ ـ ـ. . 10 
1- د.علي مجيد حمادي ـالتشابك الاقتصادي بين النظرية والتطبيق ـ دار اليازوري العلمية للنشر

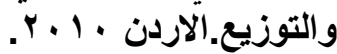

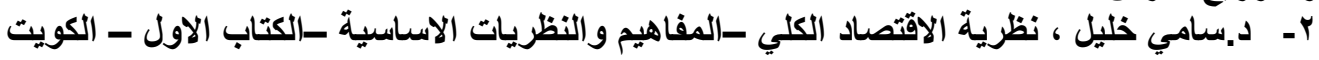

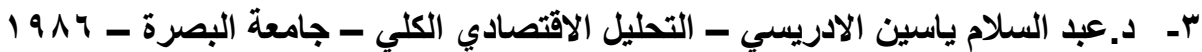

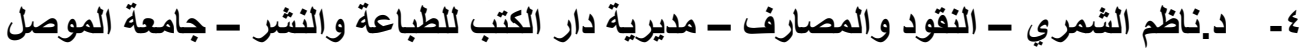

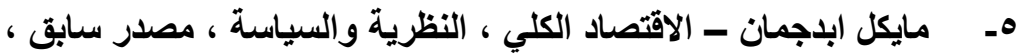

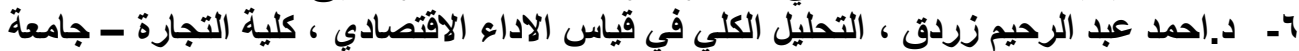

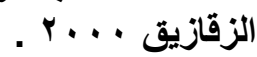

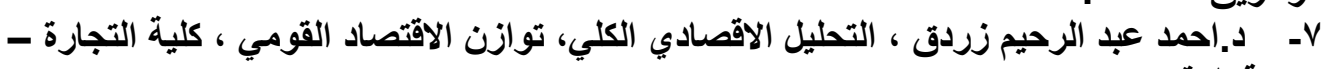

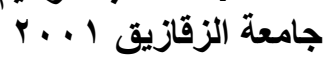

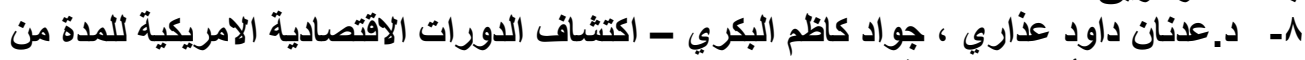

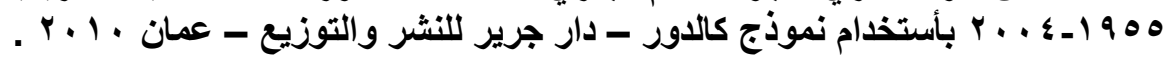

9- Nicolas Kaldor "The Irrelevance of equilibrium Economic" The Economic Journal‘1972.

10 -James R. Markusen "Introduction to Gam 5 for economic Equilibrium problem" University of Colorado، 2005

11- Elmer G. Wiens "macroeconomics Theory \& Application"working paper 2011 
التوازز الاقتصادي العام ( IS-LM )

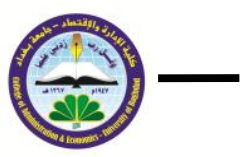

\title{
Analysis of the rebound effect and interactive between fiscal and monetary policy on the General economic equilibrium (IS-LM)
}

\begin{abstract}
Will address this research interaction and coordination between fiscal and monetary policies and the impact of this interaction and coordination on economic stability and growth' and how the financial implications of monetary policy may stimulate action monetary policy and treatment side effects and the nature of responsiveness and bounce between procedures both two policies and their impact on the balance of overall economic and explained in the folds of searchjustifications coordination and the extent necessary in order to address the imbalances in economic activity through twinning actions of monetary and fiscal has embodied this coordination and interaction between policies and their impact mutual consistent and inconsistent when studying balance general economic and pillars of balance in the money market and the balance in the market commodity (Is-Lm). The balance monetary and commodity introductions essential to balance the general economic with origin and dimension Keynesian who put its basic idea balance economists Hekes and Hansen in their analysis of the balance of Keynesian Economics (income model simplex) and then was developed model to model (Is-Lm) thanks to their contributions theory and summarized tie between aggregate demand cash and aggregate supply commodity which plays monetary policy role "influential" and sensitive "in aggregate demand cash (monetary value of goods and services) also affect fiscal policy effectively in the overall width of the commodity (content Commodity money) and both two policies by its tools and policies different leaves effects mutually responsive about the purpose or mutual feedback effects on the target ... that stable economic balance is a viable case to restore balance in the event that this balance to the imbalance by self-forces and the unstable economic balance is non-viable state to restore balance، but remain in the folds oscillate between imbalance and stabilize the rates of certain economic use and output and prices .
\end{abstract}

Key words/

The general Economic Equilibrium- Equilibrium of Monetry MarketEquilibrium of Commodity Market- stablize economic balance- The nonstablize economic balance The Elestisty of investment، Liquidity trap- The demand of money for speculitve- Expantionary \& contractionary policy. 